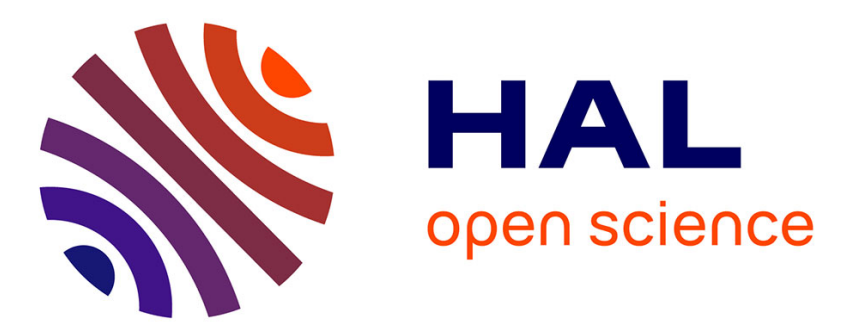

\title{
Existence and Uniqueness Theorems for the Two-Dimensional Ericksen-Leslie System
}

Gregory A. Chechkin, Tudor S. Ratiu, Maxim S. Romanov, Vyacheslav N. Samokhin

\section{- To cite this version:}

Gregory A. Chechkin, Tudor S. Ratiu, Maxim S. Romanov, Vyacheslav N. Samokhin. Existence and Uniqueness Theorems for the Two-Dimensional Ericksen-Leslie System. Journal of Mathematical Fluid Mechanics, 2016, 18 (3), pp.571-589. hal-01383845

\section{HAL Id: hal-01383845 https://hal.science/hal-01383845}

Submitted on 19 Oct 2016

HAL is a multi-disciplinary open access archive for the deposit and dissemination of scientific research documents, whether they are published or not. The documents may come from teaching and research institutions in France or abroad, or from public or private research centers.
L'archive ouverte pluridisciplinaire HAL, est destinée au dépôt et à la diffusion de documents scientifiques de niveau recherche, publiés ou non, émanant des établissements d'enseignement et de recherche français ou étrangers, des laboratoires publics ou privés. 


\title{
Existence and Uniqueness Theorems for the Two-Dimensional Ericksen-Leslie System
}

\author{
Gregory A. Chechkin, Tudor S. Ratiu, Maxim S. Romanov and Vyacheslav N. Samokhin \\ Communicated by $M$. Hieber
}

\begin{abstract}
In this paper we study the two dimensional Ericksen-Leslie equations for the nematodynamics of liquid crystals if the moment of inertia of the molecules does not vanish. We prove short time existence and uniqueness of strong solutions for the initial value problem in two situations: the space-periodic problem and the case of a bounded domain with spatial Dirichlet boundary conditions on the Eulerian velocity and the cross product of the director field with its time derivative. We also show that the speed of propagation of the director field is finite and give an upper bound for it.
\end{abstract}

Keywords. Liquid crystals, Ericksen-Leslie equations, nematodynamics, existence and uniqueness, director field, speed of propagation.

\section{Introduction}

Liquid crystals, a state of matter exhibiting properties between those of a liquid and a solid crystal, were discovered by the botanical physiologist Friedrich Reinitzer in 1888 . They were first studied by the physicist Otto Lehman around 1900 and the chemist Daniel Vorländer who, at the beginning of the 20th century, synthesized most of the liquid crystals known at the time. The mineralogist and crystallographer George Friedel gave a first classification of the liquid crystals around the same time. The first modern physical theory of liquid crystals, due to the physicist Carl Wilhelm Oseen, was formulated during the 1930s. In the same period, the physicist Victor N. Tsvetkov used viscosity, dielectric, and diamagnetic anisotropy of liquid crystals to create a general theory of the mesomorphic state. He invented methods that led to the understanding of the dynamic properties of the mesophases. In 1963, James Ferguson used liquid crystals in practical applications, such as detecting thermal fields. After that, interest in liquid crystals has increased dramatically.

The microscopic theoretical treatment of fluids is very complicated, since interactions between molecules should be taken into account. In the case of liquid crystals, which are anisotropic in all of these interactions, the analysis is another order of magnitude more complicated. However, there are a number of microscopic theories that can at least predict the general behavior of the phase transitions in liquid crystal systems (see, for instance [1]).

There are two broad kinds of liquid crystals; thermotropic (formed by heating a solid) and lyotropic (formed in mixtures of solids with solvents). The thermotropic liquid crystals may be nematic, smectic, cholesteric (see Fig. $1^{1}$ ), chiral, discotic, or in the blue phase. In all these types of liquid crystals, the orientation of the molecular dipole is defined by a unit vector, called "director".

I From Wikipedia article http://www russika ru/t.php?t=4305 and a google search for images of liquid crystals, where many more pictures of various phases of liquid crystals can be found. 

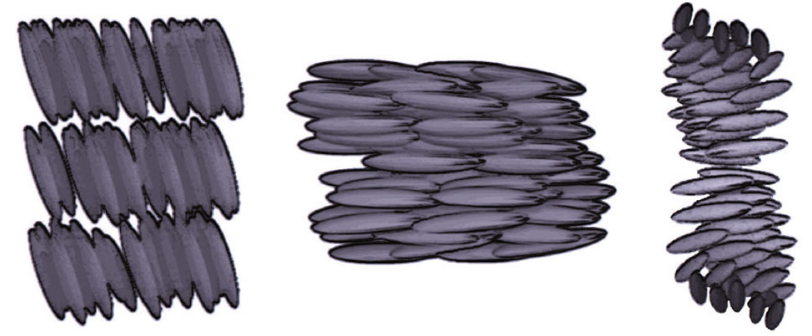

FIG. 1. The structure of smectic (left), nematic (center) and cholesteric (right) liquid crystals

In this paper, we study liquid crystal nematodynamics (i.e., the hydrodynamics of nematic liquid crystals). In previous work, we investigated periodic mesomorphic media [2] and homogenization of micro inhomogeneous nematic liquid crystals (periodic in [3] and random in [4]).

The hydrodynamic theory of liquid crystals, due to Ericksen and Leslie, was developed in the 1960's [5-8]; see also $[9,10]$. The subject of our research is the Ericksen-Leslie system describing the dynamics of nematic liquid crystals

$$
\left\{\begin{array}{l}
\dot{\mathbf{u}}-\mu \Delta \mathbf{u}=-\nabla p-\frac{\partial}{\partial x_{j}}\left(\frac{\partial \mathcal{F}}{\partial \mathbf{n}_{x_{j}}} \cdot(\nabla \mathbf{n})\right)+\mathbf{F}+f, \quad \operatorname{div} \mathbf{u}=0 \\
J \ddot{\mathbf{n}}-2 q \mathbf{n}+\mathbf{h}=g+\mathbf{G}, \quad\|\mathbf{n}\|=1,
\end{array}\right.
$$

where summation on repeated indices is understood, $\mathbf{n}_{x_{j}}:=\frac{\partial}{\partial x_{j}} \mathbf{n}$, and

$$
\frac{\partial \mathcal{F}}{\partial \mathbf{n}_{x_{j}}} \cdot(\nabla \mathbf{n}):=\frac{\partial F}{\partial n_{k, x_{j}}} \nabla n_{k}
$$

(see also [1, formulas (3.90), (3.99), (3.100), (5.2)], [11, page 90], or [12]). Here, $\mathbf{u}$ is the Eulerian, or spatial velocity vector field, $\mathbf{n}=\left(n_{1}, n_{2}, n_{3}\right)$ is the director field, the constant $\mu>0$ is the viscosity coefficient, the constant $J>0$ is the moment of inertia of the molecule, $\mathbf{F}(x, t)$ and $\mathbf{G}(x, t)$ are given external forces, and $:=\frac{\partial}{\partial t}+\mathbf{u} \cdot \nabla$ is the material derivative. The terms $f$ and $g$ correspond to the dissipative part of the stress tensor and the dissipative part of the intrinsic body force, respectively, and they depend on $\mathbf{u}, \mathbf{n}$, and their derivatives. The function $\mathcal{F}(\mathbf{n}, \nabla \mathbf{n})$ is the Oseen-Zöcher-Frank free energy and is defined by

$$
\mathcal{F}(\mathbf{n}, \nabla \mathbf{n}):=K_{1} \mathbf{n} \cdot \operatorname{curl} \mathbf{n}+\frac{1}{2}\left(K_{11}(\operatorname{div} \mathbf{n})^{2}+K_{22}(\mathbf{n} \cdot \operatorname{curl} \mathbf{n})^{2}+K_{33}\|\mathbf{n} \times \operatorname{curl} \mathbf{n}\|^{2}\right) .
$$

The molecular field $\mathbf{h}$ is defined by

$$
\mathbf{h}:=\frac{\partial \mathcal{F}}{\partial \mathbf{n}}-\frac{\partial}{\partial x_{j}}\left(\frac{\partial \mathcal{F}}{\partial \mathbf{n}_{x_{j}}}\right) .
$$

The pressure $p$ and the Lagrange multiplier $2 q$ are determined, respectively, by the conditions $\operatorname{div} \mathbf{u}=0$ and $\|\mathbf{n}\|=1$.

We are interested in the non-dissipative case, i.e., $g=\mathbf{0}, f=\mathbf{0}$. Since the liquid crystal is nematic, we necessarily have $K_{1}=0$. For simplicity, we study the one-constant approximation, i.e., we assume

$$
K_{11}=K_{22}=K_{33}=: K>0 .
$$

Thus, using $\|\mathbf{n}\|=1$, the Oseen-Zöcher-Frank free energy (2) becomes

$$
\begin{aligned}
\mathcal{F} & =\frac{K_{11}}{2}(\operatorname{div} \mathbf{n})^{2}+\frac{K_{22}-K_{33}}{2}(\mathbf{n} \cdot \operatorname{curl} \mathbf{n})^{2}+\frac{K_{33}}{2}\|\mathbf{n}\|^{2}\|\operatorname{curl} \mathbf{n}\|^{2} \\
& =\frac{K}{2}(\operatorname{div} \mathbf{n})^{2}+\frac{K}{2}\left(n_{j, x_{i}} n_{j, x_{i}}-n_{i, x_{j}} n_{j, x_{i}}\right),
\end{aligned}
$$


where the subscript $x_{j}$ means partial derivative relative to the spatial coordinate $x_{j}$ and summation on repeated indices is understood, irrespective of their position. Hence,

$$
\frac{\partial \mathcal{F}}{\partial \mathbf{n}}=0, \quad \frac{\partial \mathcal{F}}{\partial n_{i, x_{j}}}=K(\operatorname{div} \mathbf{n}) \delta_{i j}+K n_{i, x_{j}}-K n_{j, x_{i}} .
$$

Lemma 1.1. The non-dissipative (i.e., $f=\mathbf{0}, g=\mathbf{0}$ ) nematic (i.e., $K_{1}=0$ ) Ericksen-Leslie equations in the one constant approximation (i.e., $K:=K_{11}=K_{22}=K_{33}>0$ ) are

$$
\left\{\begin{array}{l}
\dot{\mathbf{u}}-\mu \Delta \mathbf{u}=-\nabla p^{\prime}-K\left(\mathbf{n}_{x_{j}} \cdot(\nabla \mathbf{n})\right)_{x_{j}}+\mathbf{F}, \quad \operatorname{div} \mathbf{u}=0, \\
J \ddot{\mathbf{n}}+\left(J\|\dot{\mathbf{n}}\|^{2}-\mathbf{h} \cdot \mathbf{n}+\mathbf{G} \cdot \mathbf{n}\right) \mathbf{n}+\mathbf{h}=\mathbf{G}, \quad\|\mathbf{n}\|=1
\end{array}\right.
$$

where the molecular field has the expression

$$
\mathbf{h}=\frac{\partial \mathcal{F}}{\partial \mathbf{n}}-\frac{\partial}{\partial x_{j}}\left(\frac{\partial \mathcal{F}}{\partial \mathbf{n}_{x_{j}}}\right)=-K \Delta \mathbf{n} .
$$

and $p^{\prime}:=p+\frac{K}{2}\left((\operatorname{div} \mathbf{n})^{2}-n_{i, x_{j}} n_{j, x_{i}}\right)$.

Proof. Formula (5) implies

$$
\begin{aligned}
\frac{1}{K} \frac{\partial}{\partial x_{j}}\left(\frac{\partial \mathcal{F}}{\partial \mathbf{n}_{x_{j}}} \cdot(\nabla \mathbf{n})\right) & =\left(\left((\operatorname{div} \mathbf{n}) \delta_{i j}+n_{i, x_{j}}-n_{j, x_{i}}\right) \nabla n_{i}\right)_{x_{j}} \\
& =\left(n_{k, x_{k} x_{j}} \nabla n_{j}+n_{k, x_{k}} \nabla n_{j, x_{j}}+\left(n_{i, x_{j}} \nabla n_{i}\right)_{x_{j}}-n_{j, x_{j} x_{i}} \nabla n_{i}-n_{j, x_{i}} \nabla n_{i, x_{j}}\right) \\
& =\left(n_{i, x_{j}} \nabla n_{i}\right)_{x_{j}}+\frac{1}{2} \nabla\left((\operatorname{div} \mathbf{n})^{2}-n_{i, x_{j}} n_{j, x_{i}}\right)
\end{aligned}
$$

and hence the first equation in (1) becomes the first equation in (6). In addition, (3) and (5) yield

$$
h_{i}=-K\left(n_{k, x_{k} x_{j}} \delta_{i j}+n_{i, x_{j} x_{j}}-n_{j, x_{i} x_{j}}\right)=-K \Delta n_{i} .
$$

which is (7).

Next, taking the dot product of the second equation in (1) with $\mathbf{n}$ yields

$$
2 q=J \ddot{\mathbf{n}} \cdot \mathbf{n}+\mathbf{h} \cdot \mathbf{n}-\mathbf{G} \cdot \mathbf{n}=-J\|\dot{\mathbf{n}}\|^{2}+\mathbf{h} \cdot \mathbf{n}-\mathbf{G} \cdot \mathbf{n}
$$

since $\mathbf{n} \cdot \dot{\mathbf{n}}=0$. Therefore, the second equation in (1) becomes

$$
0=J \ddot{\mathbf{n}}-2 q \mathbf{n}+\mathbf{h}-\mathbf{G}=J \ddot{\mathbf{n}}+\left(J\|\dot{\mathbf{n}}\|^{2}-\mathbf{h} \cdot \mathbf{n}+\mathbf{G} \cdot \mathbf{n}\right) \mathbf{n}+\mathbf{h}-\mathbf{G}
$$

which is the second equation in (6).

We rewrite the Ericksen-Leslie equations (6) as a system of three equations of first order by introducing a new vector field $\boldsymbol{\nu}$. This vector field appeared for the first time in [13], motivated by the geometric and variational structure of the conservative version of the Ericksen-Leslie equations (i.e., $\mu=0, f=g=\mathbf{0}$, $\mathbf{F}=\mathbf{G}=\mathbf{0})$.

Theorem 1.1. If $(\mathbf{u}, \mathbf{n})$ is a solution of Ericksen-Leslie equations (6), $\|\mathbf{n}\|=1$, define the vector field $\boldsymbol{\nu}:=\mathbf{n} \times \dot{\mathbf{n}}$. Then, $(\mathbf{u}, \boldsymbol{\nu}, \mathbf{n})$ is a solution of the system

$$
\begin{aligned}
\dot{\mathbf{u}}-\mu \Delta \mathbf{u} & =-\nabla p^{\prime}-\left(K n_{k, x_{j}} \nabla n_{k}\right)_{x_{j}}+\mathbf{F}, \quad \operatorname{div} \mathbf{u}=0, \\
J \dot{\boldsymbol{\nu}} & =-K(\Delta \mathbf{n}) \times \mathbf{n}+\mathbf{n} \times \mathbf{G}, \\
\dot{\mathbf{n}} & =\boldsymbol{\nu} \times \mathbf{n},
\end{aligned}
$$

with initial conditions $\mathbf{n}_{0}$ and $\boldsymbol{\nu}_{0}$ satisfying $\left\|\mathbf{n}_{0}\right\|=1$ and $\mathbf{n}_{0} \cdot \boldsymbol{\nu}_{0}=0$.

Conversely, if $(\mathbf{u}, \boldsymbol{\nu}, \mathbf{n})$ is a solution of the system (9)-(11) with initial conditions $\mathbf{n}_{0}$ and $\boldsymbol{\nu}_{0}$ satisfying $\left\|\mathbf{n}_{0}\right\|=1$ and $\mathbf{n}_{0} \cdot \boldsymbol{\nu}_{0}=0$, then $(\mathbf{u}, \mathbf{n})$ is a solution of the Ericksen-Leslie equations (6). 
Proof. Suppose that $(\mathbf{u}, \mathbf{n})$ is a solution of Ericksen-Leslie equations (6) and define the vector field $\boldsymbol{\nu}:=\mathbf{n} \times \dot{\mathbf{n}}$. Then, $\boldsymbol{\nu} \times \mathbf{n}=(\mathbf{n} \times \dot{\mathbf{n}}) \times \mathbf{n}=\dot{\mathbf{n}}$ since $\|\mathbf{n}\|=1$ and $\mathbf{n} \cdot \dot{\mathbf{n}}=0$, which gives (11). The first equation in (6) coincides with (9). Finally,

$$
J \dot{\boldsymbol{\nu}}=J(\mathbf{n} \times \dot{\mathbf{n}}) \dot{n} \times \mathbf{n} \ddot{\mathbf{n}} \stackrel{(6)}{=}-\mathbf{n} \times \mathbf{h}+\mathbf{n} \times \mathbf{G} \stackrel{(7)}{=}-K(\Delta \mathbf{n}) \times \mathbf{n}+\mathbf{n} \times \mathbf{G}
$$

which is (10).

Since $(\mathbf{u}, \mathbf{n})$ is a solution of (6), we have $\|\mathbf{n}\|=1$. In addition, $\mathbf{n} \cdot \boldsymbol{\nu}=\mathbf{n} \cdot(\mathbf{n} \times \dot{\mathbf{n}})=0$. Therefore, the initial conditions of the system (9)-(11) necessarily satisfy $\left\|\mathbf{n}_{0}\right\|=1$ and $\mathbf{n}_{0} \cdot \boldsymbol{\nu}_{0}=0$, as stated.

Conversely, suppose that $(\mathbf{u}, \boldsymbol{\nu}, \mathbf{n})$ is a solution of the system (9)-(11) with initial conditions satisfying $\left\|\mathbf{n}_{0}\right\|=1$ and $\mathbf{n}_{0} \cdot \boldsymbol{\nu}_{0}=0$. Then $\left(\|\mathbf{n}\|^{2}\right)^{\cdot}=2 \mathbf{n} \cdot \dot{\mathbf{n}} \stackrel{(11)}{=} 2 \mathbf{n} \cdot(\boldsymbol{\nu} \times \mathbf{n})=0$ and

$$
(\mathbf{n} \cdot \boldsymbol{\nu}) \dot{=} \cdot \boldsymbol{\nu}+\mathbf{n} \cdot \dot{\boldsymbol{\nu}}=(\boldsymbol{\nu} \times \mathbf{n}) \cdot \mathbf{n}+\mathbf{n} \cdot \frac{1}{J}(-K \Delta \mathbf{n} \times \mathbf{n}+\mathbf{n} \times \mathbf{G})=0
$$

by (10) and (11), which implies $\|\mathbf{n}\|=1$ and $\mathbf{n} \cdot \boldsymbol{\nu}=0$.

We have $\mathbf{n} \times \dot{\mathbf{n}} \stackrel{(11)}{=} \mathbf{n} \times(\boldsymbol{\nu} \times \mathbf{n})=\boldsymbol{\nu}$ since $\|\mathbf{n}\|=1$ and $\mathbf{n} \cdot \boldsymbol{\nu}=0$. Conversely, if $\boldsymbol{\nu}=\mathbf{n} \times \dot{\mathbf{n}}$, then $\boldsymbol{\nu} \times \mathbf{n}=(\mathbf{n} \times \dot{\mathbf{n}}) \times \mathbf{n}=\dot{\mathbf{n}}$, since $\|\mathbf{n}\|=1$.

The first equation in (6) coincides with (9).

Finally, by (7), (11), $\|\mathbf{n}\|=1$, and $\boldsymbol{\nu} \cdot \mathbf{n}=0$, we get

$$
\begin{aligned}
J \ddot{\mathbf{n}}+ & \left(J\|\dot{\mathbf{n}}\|^{2}-\mathbf{h} \cdot \mathbf{n}+\mathbf{G} \cdot \mathbf{n}\right) \mathbf{n}+\mathbf{h}=J \dot{\boldsymbol{\nu}} \times \mathbf{n}+J \boldsymbol{\nu} \times \dot{\mathbf{n}}+\left(J\|\dot{\mathbf{n}}\|^{2}+K \Delta \mathbf{n} \cdot \mathbf{n}+\mathbf{G} \cdot \mathbf{n}\right) \mathbf{n}-K \Delta \mathbf{n} \\
& (10) \\
= & -K((\Delta \mathbf{n}) \times \mathbf{n}) \times \mathbf{n}+(\mathbf{n} \times \mathbf{G}) \times \mathbf{n}+J \boldsymbol{\nu} \times(\boldsymbol{\nu} \times \mathbf{n}) \\
& +\left(J\|\boldsymbol{\nu}\|^{2}+K \Delta \mathbf{n} \cdot \mathbf{n}+\mathbf{G} \cdot \mathbf{n}\right) \mathbf{n}-K \Delta \mathbf{n} \\
= & K \Delta \mathbf{n}-K(\mathbf{n} \cdot \Delta \mathbf{n}) \mathbf{n}+\mathbf{G}-(\mathbf{G} \cdot \mathbf{n}) \mathbf{n}-J\|\boldsymbol{\nu}\|^{2} \mathbf{n} \\
& +J\|\boldsymbol{\nu}\|^{2} \mathbf{n}+K(\Delta \mathbf{n} \cdot \mathbf{n}) \mathbf{n}+(\mathbf{G} \cdot \mathbf{n}) \mathbf{n}-K \Delta \mathbf{n}=\mathbf{G}
\end{aligned}
$$

which is the second equation in (6).

In terms of $\boldsymbol{\nu}$, by (8) and (11), we have $2 q=\mathbf{h} \cdot \mathbf{n}-J\|\boldsymbol{\nu}\|^{2}-\mathbf{G} \cdot \mathbf{n}$.

Because of Theorem 1.1, we work in the rest of the paper exclusively with the Ericksen-Leslie equations expressed as (9)-(11) with initial conditions $\left(\mathbf{u}_{0}, \boldsymbol{\nu}_{0}, \mathbf{n}_{0}\right)$ satisfying $\left\|\mathbf{n}_{0}\right\|=1$ and $\mathbf{n}_{0} \cdot \boldsymbol{\nu}_{0}=0$.

Since the structure of the full Ericksen-Leslie system is sufficiently complicated, simplified models have been introduced in order to get some reasonable results. Most of the known results are obtained under the assumption $J=0$. For the incompressible model in [14], Lin introduced a simplification of the general Ericksen-Leslie system that keeps many of the mathematical difficulties of the original system by using a Ginzburg-Landau approximation to relax the nonlinear constraint. Namely, instead of the restriction $\|\mathbf{n}\|=1$, the penalty term $\frac{1}{\epsilon^{2}}\left(\|\mathbf{n}\|^{2}-1\right)^{2}$ was added to the free energy functional. In [15], Lin and Liu showed the global existence of weak solutions and smooth solutions for that approximation. In [16], a very simple proof of local well-posedness for this coupled system was provided using a contraction mapping argument. It was proved that this system is globally well-posed and has compact global attractors in 2D. Recently, Hong [17] and Lin-Liu-Wang [18] showed, independently, the global existence of weak solutions of an incompressible model in two dimensional space. Moreover, in [18], the regularity of solutions, except for a countable set of singularities whose projection on the time axis is a finite set, has been obtained (see also [19]). In [20], Wang established a global well-posedness theory for the incompressible liquid crystals for rough initial data. A simplified Ericksen-Leslie system for two-dimensional compressible flow was considered in [21]. Hieber, Nesensohn, Prüss, and Schade analyzed in [22] the simplified system as a parabolic evolutional equation in an $L_{p}-L_{q}$-setting and studied the system near an equilibrium. 
In the present paper we focus on the system (9)-(11) in the hyperbolic case $J>0$ and prove an existence and uniqueness theorem for two-dimensional periodic media as well as for the problem in a bounded domain of the plane. We also prove the finite propagation speed of waves in such media.

Some results in this paper were announced in [23].

\section{Two-Dimensional Solution in a Periodic Domain}

Consider a liquid crystal flow in $\mathbb{R}^{3}$. The flow is called two-dimensional if all unknowns in the EricksenLeslie system are independent of the third coordinate $x_{3}$; so we can suppose that they are all defined on a plane $\left(x_{1}, x_{2}\right)$.

Let $Q_{T}:=(0, T) \times \mathbb{T}$, where $\mathbb{T}:=\mathbb{R}^{2} / \mathbb{Z}^{2}$ is the two-dimensional flat torus. We study the system (9)-(11) in $Q_{T}$ with initial conditions

$$
\mathbf{u}(0, x)=\mathbf{u}_{0}, \quad \boldsymbol{\nu}(0, x)=\boldsymbol{\nu}_{0}, \quad \mathbf{n}(0, x)=\mathbf{n}_{0},
$$

satisfying $\left\|\mathbf{n}_{0}\right\|=1$ and $\mathbf{n}_{0} \cdot \boldsymbol{\nu}_{0}=0$. Here $\mathbf{u}, \boldsymbol{\nu}, \mathbf{n}$ are unknown vector fields, $p^{\prime}$ is an unknown scalar function, and $J, K, \mu$ are fixed strictly positive numbers. The material derivative $f_{t}+\sum_{i=1}^{3} u^{i} f_{x_{i}}$ turns into $f_{t}+\sum_{i=1}^{2} u^{i} f_{x_{i}}$.

It is natural to consider a flat motion, i.e., $\mathbf{u}: \mathbb{T} \rightarrow \mathbb{R}^{2} \times\left\{u^{3}=0\right\}$. Note that the vector fields $\mathbf{n}, \boldsymbol{\nu}$ are always three-dimensional, even if they are defined on a flat two-dimensional domain; in particular the director field is not necessarily tangential to the plane $\left\{x_{3}=0\right\}$.

\subsection{Notations and Definitions}

Throughout the paper we use the following notations:

- $\dot{f}:=\frac{\partial f}{\partial t}+\mathbf{u} \cdot \nabla f=f_{t}+u^{j} f_{x_{j}}$ is the material time derivative of $f$;

- a bold letter $\mathbf{b}$ denotes a 3 -dimensional vector $\mathbf{b}=\left(b^{1}, b^{2}, b^{3}\right)$, or a vector field with values in $\mathbb{R}^{3}$;

- standard summation convention is used on repeated indices, independent on their position, e.g., $a_{i} b_{i}:=\sum_{i} a_{i} b_{i}$

- $L_{2}(\mathbb{T}):=\left\{\mathbf{v}: \mathbb{T} \rightarrow \mathbb{R}^{3} \mid \int_{\mathbb{T}}\|\mathbf{v}\|^{2} d^{2} x<\infty\right\}$;

- $W_{2}^{m}(\mathbb{T})$ is the Sobolev space of functions on $\mathbb{T}$ having $m$ distributional derivatives in $L_{2}(\mathbb{T})$;

- $\operatorname{Sol}(\mathbb{T}):=\left\{\mathbf{v}: \mathbb{T} \rightarrow \mathbb{R}^{3} \mid \mathbf{v} \in C^{\infty}(\mathbb{T}), \operatorname{div} \mathbf{v}=0\right\}$

- $\operatorname{Sol}\left(Q_{T}\right):=\left\{\mathbf{v} \in C^{\infty}\left(Q_{T}\right) \mid \mathbf{v}(t, \cdot) \in \operatorname{Sol}(\mathbb{T}), \forall t \in(0, T)\right\}$;

- $\operatorname{Sol}_{2}(\mathbb{T})$ is the closure of $\operatorname{Sol}(\mathbb{T})$ in the norm $L_{2}(\mathbb{T})$;

- $\operatorname{Sol}_{2}^{m}(\mathbb{T})$ is the closure of $\operatorname{Sol}(\mathbb{T})$ in the norm $W_{2}^{m}(\mathbb{T})$.

Definition 2.1. A quadruple $\left(\mathbf{u}, \boldsymbol{\nu}, \mathbf{n}, \nabla p^{\prime}\right)$ is a strong solution of problem (9)-(12) in the domain $Q_{T}$ if

(i) $\mathbf{u}$ is a time-dependent vector field in $L_{2}\left((0, T) ; S o l_{2}^{3}(\mathbb{T})\right), \mathbf{u}_{t} \in L_{2}\left(Q_{T}\right)$;

(ii) $\boldsymbol{\nu}$ is a vector field in $L_{\infty}\left((0, T) ; W_{2}^{2}(\mathbb{T})\right), \boldsymbol{\nu}_{t} \in L_{\infty}\left((0, T) ; L_{2}(\mathbb{T})\right)$;

(iii) $\mathbf{n}$ is a vector field in $L_{\infty}\left((0, T) ; W_{2}^{3}(\mathbb{T})\right), \mathbf{n}_{t} \in L_{\infty}\left((0, T) ; W_{2}^{1}(\mathbb{T})\right)$;

(iv) $\nabla p^{\prime} \in L_{2}\left(Q_{T}\right)$;

(v) $\mathbf{u}, \mathbf{n}, \boldsymbol{\nu}$ satisfy the initial conditions (12), i.e., $(\mathbf{u}, \mathbf{n}, \boldsymbol{\nu}) \rightarrow\left(\mathbf{u}_{0}, \mathbf{n}_{0}, \boldsymbol{\nu}_{0}\right)$ in $L_{2}(\mathbb{T})$ as $t \rightarrow 0$;

(vi) Equations (9)-(11) hold almost everywhere.

The first goal of the paper is to prove existence of strong solutions to the problem (9)-(12).

Theorem 2.1. Let $\mathbf{F}=0, \mathbf{G}=0$. Suppose $\mathbf{u}_{0} \in \operatorname{Sol}_{2}^{2}(\mathbb{T}), \Delta \boldsymbol{\nu}_{0} \in L_{2}(\mathbb{T}), \Delta \mathbf{n}_{0} \in W_{2}^{1}(\mathbb{T})$. Then there is a $T>0$ such that the solution to problem (9)-(12) (as given in Definition 2.1) does exist.

The proof of this theorem is given in the next subsections. 


\subsection{Galerkin-Type Approximations}

We begin the proof with a classical approximation method.

Select two sequences of subspaces $E^{1} \subset E^{2} \subset \cdots$ and $F^{1} \subset F^{2} \subset \cdots$ such that $\cup_{k \in \mathbb{N}} E^{k}$ is dense in $\operatorname{Sol}_{2}^{2}(\mathbb{T})$ and $\cup_{k \in \mathbb{N}} F^{k}$ is dense in $W_{2}^{2}(\mathbb{T})$ (and, consequently, in $W_{2}^{1}(\mathbb{T})$ ).

Since the inclusions $W_{2}^{1}(\mathbb{T}) \hookrightarrow L_{2}(\mathbb{T})$ and $\operatorname{Sol}_{2}^{1}(\mathbb{T}) \hookrightarrow \operatorname{Sol}_{2}(\mathbb{T})$ are both compact, the operator $\Delta$ : $\operatorname{Sol}(\mathbb{T}) \rightarrow \operatorname{Sol}(\mathbb{T})$ is a symmetric operator that extends to a self-adjoint operator on $S o l_{2}(\mathbb{T})$ and $L_{2}(\mathbb{T})$. Fix bases of eigenfunctions of $\Delta$ in $S_{0}(\mathbb{T})$ and $L_{2}(\mathbb{T})$ and denote by $E^{k}$ and $F^{k}$ the linear span of the first $k$ eigenfunctions in $S_{2} l_{2}(\mathbb{T})$ and $L_{2}(\mathbb{T})$, respectively. The subspaces $E^{k}$ and $F^{k}$ consist of smooth functions and thus $\cup_{k \in \mathbb{N}} E^{k}$ and $\cup_{k \in \mathbb{N}} F^{k}$ are dense in $S o l_{2}^{2}(\mathbb{T})$ and $W_{2}^{2}(\mathbb{T})$, respectively.

Proposition 2.1. Let $\left(\mathbf{u}_{0 k}, \boldsymbol{\nu}_{0, k}, \mathbf{n}_{0, k}\right) \in E^{k} \times F^{k} \times F^{k}$ be an approximation of the initial data $\left(\mathbf{u}_{0}, \boldsymbol{\nu}_{0}, \mathbf{n}_{0}\right)$ for fixed $k$.

Then, for some $T>0$, there exists a solution $\left(\mathbf{u}_{k}, \boldsymbol{\nu}_{k}, \mathbf{n}_{k}\right) \subset C^{0}\left((0, T) ; E^{k} \times F^{k} \times F^{k}\right)$ of the problem

$$
\begin{gathered}
\left(\mathbf{u}_{k, t}, \boldsymbol{\omega}\right)=-\left(u_{k}^{l} \mathbf{u}_{k, x_{l}}, \boldsymbol{\omega}\right)+\mu\left(\Delta \mathbf{u}_{k}, \boldsymbol{\omega}\right)-K\left(\Delta \mathbf{n}_{k} \cdot \nabla \mathbf{n}_{k}, \boldsymbol{\omega}\right), \\
J\left(\boldsymbol{\nu}_{k, t}, \boldsymbol{\zeta}\right)+\left(J u_{k}^{l} \boldsymbol{\nu}_{k, x_{l}}, \boldsymbol{\zeta}\right)+K\left(\Delta \mathbf{n}_{k} \times \mathbf{n}_{k}, \boldsymbol{\zeta}\right)=0 \\
\left(\mathbf{n}_{k, t}, \boldsymbol{\psi}\right)+\left(u_{k}^{l} \mathbf{n}_{k, x_{l}}, \boldsymbol{\psi}\right)-\left(\boldsymbol{\nu}_{k} \times \mathbf{n}_{k}, \boldsymbol{\psi}\right)=0 \\
\left.\left(\mathbf{u}_{k}, \boldsymbol{\nu}_{k}, \mathbf{n}_{k}\right)\right|_{t=0}=\left(\mathbf{u}_{0 k}, \boldsymbol{\nu}_{0 k}, \mathbf{n}_{0 k}\right)
\end{gathered}
$$

where the identities above hold for all $\boldsymbol{\omega} \in E^{k}, \boldsymbol{\zeta}, \boldsymbol{\psi} \in F^{k}$, and $(\mathbf{u}, \mathbf{v})=\int_{\mathbb{T}} \mathbf{u} \cdot \mathbf{v} d x_{1} d x_{2}$ is the $L_{2}(\mathbb{T})$-inner product.

Indeed, the system (13)-(16) could be regarded as a Cauchy problem for the ordinary differential equation $X_{t}=f(X)$ in $3 k$-dimensional space with continuous right-hand side. Due to the Cauchy-Peano theorem, there exists some small $T_{0}>0$ such that this problem has a solution for $|t|<T_{0}=: T$.

Remark 2.1. If $u_{0 k}^{3}=0$, then for any $t<T_{0}$, we have $u_{k}^{3}(t)=0$.

The next step is to get a uniform estimate on $\left(\mathbf{u}_{k}, \boldsymbol{\nu}_{k}, \mathbf{n}_{k}\right)$ in some appropriate norm.

\subsection{Energy Conservation}

We need the following identity.

Lemma 2.1. For all $t \in(0, T)$ we have

$$
\begin{aligned}
\int_{\mathbb{T}}\left(\left\|\mathbf{u}_{k}(t)\right\|^{2}+J\left\|\boldsymbol{\nu}_{k}(t)\right\|^{2}+K\left\|\nabla \mathbf{n}_{k}(t)\right\|^{2}\right) d x_{1} d x_{2}+2 \mu \int_{0}^{t} \int_{\mathbb{T}}\left\|\nabla \mathbf{u}_{k}\right\|^{2} d x_{1} d x_{2} d t \\
=\int_{\mathbb{T}}\left(\left\|\mathbf{u}_{0 k}\right\|^{2}+\left\|\boldsymbol{\nu}_{0 k}\right\|^{2}+\left\|\nabla \mathbf{n}_{0 k}\right\|^{2}\right) d x_{1} d x_{2} .
\end{aligned}
$$

Proof. In this proof we use $(\mathbf{u}, \boldsymbol{\nu}, \mathbf{n})$ instead of $\left(\mathbf{u}_{k}, \boldsymbol{\nu}_{k}, \mathbf{n}_{k}\right)$, in order to simplify notation.

In Eqs. (13)-(15) we substitute $(\boldsymbol{\omega}, \boldsymbol{\zeta}, \boldsymbol{\psi})=(\mathbf{u}, \boldsymbol{\nu},-K \Delta \mathbf{n})$. Since $\Delta: F^{k} \rightarrow F^{k}$, this substitution is allowed. Taking the integral over the interval $(0, t)$ in $(13)$ and using periodicity of all functions, we obtain

$$
\left.\int_{\mathbb{T}} \frac{1}{2}\|\mathbf{u}\|^{2}\right|_{0} ^{t} d x_{1} d x_{2}=\int_{0}^{t} \int_{\mathbb{T}}\left(-\mu\|\nabla \mathbf{u}\|^{2}+K n_{x_{j}}^{s} n_{x_{i}}^{s} u_{x_{j}}^{i}\right) d x_{1} d x_{2} d t .
$$


The sum of the integrals over the interval $(0, t)$ of the relations $(14)$ and $(15)$ reads

$$
\begin{aligned}
0= & \int_{0}^{t} \int_{\mathbb{T}} J\left(\boldsymbol{\nu} \cdot \boldsymbol{\nu}_{t}+u^{l} \nu_{x_{l}}^{i} \nu^{i}\right) \\
& +K\left((\Delta \mathbf{n} \times \mathbf{n}) \cdot \boldsymbol{\nu}+(\boldsymbol{\nu} \times \mathbf{n}) \cdot \Delta \mathbf{n}-n_{t}^{i} n_{x_{s} x_{s}}^{i}-u^{j} n_{x_{j}}^{i} n_{x_{s} x_{s}}^{i}\right) d x_{1} d x_{2} d t \\
= & \left.\int_{\mathbb{T}} \frac{1}{2}\left(J\|\boldsymbol{\nu}\|^{2}+K n_{x_{s}}^{i} n_{x_{s}}^{i}\right) d x_{1} d x_{2}\right|_{0} ^{t}+\int_{0}^{t} \int_{\mathbb{T}} K u_{x_{s}}^{j} n_{x_{s}}^{i} n_{x_{j}}^{i} d x_{1} d x_{2} d t
\end{aligned}
$$

Taking the sum of (17) and (18) we obtain

$$
\left.\frac{1}{2} \int_{\mathbb{T}}\left(\|\mathbf{u}\|^{2}+J\|\boldsymbol{\nu}\|^{2}+K\|\nabla \mathbf{n}\|^{2}\right) d x_{1} d x_{2}\right|_{0} ^{t}+\mu \int_{0}^{t} \int_{\mathbb{T}}\|\nabla \mathbf{u}\|^{2} d x_{1} d x_{2} d t=0,
$$

which proves the stated identity.

Corollary 1. Problem (13)-(16) has a solution for every $T>0$.

Proof. Reconsider our problem as an ordinary differential equation $X_{t}=f(X)$ with continuous right hand side.

The value $T_{0}$ obtained from the Cauchy-Peano theorem depends only on the function $f$ and on the norm of the initial data. Since $f$ doesn't depend on $t$ and for any $t \in\left(0, T_{0}\right)$ the solution $X(t)=$ $\left(\mathbf{u}_{k}(t), \boldsymbol{\nu}_{k}(t), \mathbf{n}_{k}(t)\right)$ is bounded with $X(0)=\left(\mathbf{u}_{k}(0), \boldsymbol{\nu}_{k}(0), \mathbf{n}_{k}(0)\right)$, the Cauchy-Peano theorem guarantees the existence of the solution on the interval $\left(t, t+T_{0}\right)$ for any $t<T_{0}$, and, consequently, on the interval $\left(0,2 T_{0}\right)$. Repeating the procedure, this proves the existence of the solution for any $t \in\left(0, N T_{0}\right)$, where $N$ is an arbitrary natural number.

\subsection{Estimates on Higher Derivatives}

Unfortunately, the results of Lemma 2.1 are not sufficient to prove the convergence of $\left(\mathbf{u}_{k}, \boldsymbol{\nu}_{k}, \mathbf{n}_{k}\right)$ to the solution of (9)-(12). We need more precise estimates.

Theorem 2.2. There exists $T>0$ and $C>0$, depending only on the initial data, and constants $J, K$, $\mu>0$, such that

$$
\begin{gathered}
\left\|\mathbf{u}_{k}\right\|_{L_{2}\left((0, T) ; W_{2}^{3}\right)},\left\|\mathbf{u}_{k}\right\|_{L_{\infty}\left((0, T) ; W_{2}^{2}\right)},\left\|\boldsymbol{\nu}_{k}\right\|_{L_{\infty}\left((0, T) ; W_{2}^{2}\right)},\left\|\mathbf{n}_{k}\right\|_{L_{\infty}\left((0, T) ; W_{2}^{3}\right)} \leq C, \\
\left\|\mathbf{u}_{k, t}\right\|_{L_{2}\left(Q_{T}\right)},\left\|\boldsymbol{\nu}_{k, t}\right\|_{L_{2}\left(Q_{T}\right)},\left\|\nabla \mathbf{n}_{k, t}\right\|_{L_{2}\left(Q_{T}\right)} \leq C .
\end{gathered}
$$

Proof. We begin by proving the first set of inequalities. In equation (14), set $\boldsymbol{\zeta}=-\Delta \boldsymbol{\nu}$ and integrate over the domain $(0, T)$ using Green's identities. We have

$$
\begin{aligned}
\left.\frac{J}{2} \int_{\mathbb{T}}\|\nabla \boldsymbol{\nu}\|^{2} d x_{1} d x_{2}\right|_{0} ^{T}= & \int_{Q_{T}}\left(-\frac{J}{2} u_{x_{k}}^{j} \nu_{x_{j}}^{i} \nu_{x_{k}}^{i}-K\left(\Delta \mathbf{n} \times \mathbf{n}_{x_{k}}\right) \cdot \boldsymbol{\nu}_{x_{k}}\right. \\
& \left.-K\left(\Delta \mathbf{n}_{x_{k}} \times \mathbf{n}\right) \cdot \boldsymbol{\nu}_{x_{k}}\right) d x_{1} d x_{2} d t .
\end{aligned}
$$

The integral of (15) with $\boldsymbol{\psi}=K \Delta^{2} \mathbf{n}$ can be written as

$$
\begin{aligned}
& \left.\frac{K}{2} \int_{\mathbb{T}} \mathbf{n}_{x_{k} x_{j}} \cdot \mathbf{n}_{x_{k} x_{j}} d x_{1} d x_{2}\right|_{0} ^{T} \\
& \quad=K \int_{Q_{T}}\left(u^{j} n_{x_{k} x_{j}}^{i} \Delta n_{x_{k}}^{i}+u_{x_{k}}^{j} n_{x_{j}}^{i} \Delta n_{x_{k}}^{i}-\left(\boldsymbol{\nu} \times \mathbf{n}_{x_{k}}+\boldsymbol{\nu}_{x_{k}} \times \mathbf{n}\right) \cdot \Delta \mathbf{n}_{x_{k}}\right) d x_{1} d x_{2} d t \\
& \quad=K \int_{Q_{T}}\left(-2 u_{x_{k}}^{j} \mathbf{n}_{x_{j} x_{k}} \cdot \Delta \mathbf{n}-\Delta u^{j} \mathbf{n}_{x_{j}} \cdot \Delta \mathbf{n}+\left(\boldsymbol{\nu}_{x_{k}} \times \mathbf{n}_{x_{k}}\right) \cdot \Delta \mathbf{n}+\left(\mathbf{n} \times \boldsymbol{\nu}_{x_{k}}\right) \cdot \Delta \mathbf{n}_{x_{k}}\right) d x_{1} d x_{2} d t .
\end{aligned}
$$


To estimate the second derivatives of $\mathbf{u}$, we set $\boldsymbol{\omega}=-\Delta \mathbf{u}$ in (13) and integrate over $(0, T)$ :

$$
\begin{aligned}
& \left.\frac{1}{2} \int_{\mathbb{T}}\|\nabla \mathbf{u}\|^{2} d x_{1} d x_{2}\right|_{0} ^{T}+\mu \int_{Q_{T}}\|\Delta \mathbf{u}\|^{2} d x_{1} d x_{2} d t \\
& \quad \stackrel{(3))}{=} \int_{Q_{T}}\left(-u_{x_{k}}^{j} \mathbf{u}_{x_{j}} \cdot \mathbf{u}_{x_{k}}+K \mathbf{n}_{x_{i}} \cdot \Delta \mathbf{n} \Delta u^{i}\right) d x_{1} d x_{2} d t .
\end{aligned}
$$

The sum of $(19)-(21)$ is

$$
\begin{aligned}
\frac{1}{2} \int_{\mathbb{T}} & \left.\left(J\|\nabla \boldsymbol{\nu}\|^{2}+K \sum_{j, k=1}^{2}\left\|\mathbf{n}_{x_{j} x_{k}}\right\|^{2}+\|\nabla \mathbf{u}\|^{2}\right) d x_{1} d x_{2}\right|_{0} ^{T}+\mu \int_{Q_{T}}\|\Delta \mathbf{u}\|^{2} d x_{1} d x_{2} d t \\
= & \int_{Q_{T}}\left(-J u_{x_{k}}^{j} \boldsymbol{\nu}_{x_{j}} \cdot \boldsymbol{\nu}_{x_{k}}-2 K u_{x_{k}}^{j} \mathbf{n}_{x_{j} x_{k}} \cdot \Delta \mathbf{n}+K\left(\boldsymbol{\nu}_{x_{k}} \times \mathbf{n}_{x_{k}}\right) \cdot \Delta \mathbf{n}\right. \\
& \left.\quad-u_{x_{k}}^{j} \mathbf{u}_{x_{j}} \cdot \mathbf{u}_{x_{k}}\right) d x_{1} d x_{2} d t .
\end{aligned}
$$

We estimate the integral on the right-hand side:

$$
\begin{gathered}
\left.\frac{1}{2} \int_{\mathbb{T}}\left(J\|\nabla \boldsymbol{\nu}\|^{2}+K \sum_{j, k=1}^{2}\left\|\mathbf{n}_{x_{j} x_{k}}\right\|^{2}+\|\nabla \mathbf{u}\|^{2}\right) d x_{1} d x_{2}\right|_{0} ^{T}+\mu \int_{Q_{T}}\|\Delta \mathbf{u}\|^{2} d x_{1} d x_{2} d t \\
\leq C_{1} \int_{0}^{T}\left(\operatorname{esssup}\|\nabla \mathbf{u}(t)\|\left(\|\nabla \boldsymbol{\nu}(t)\|_{L_{2}(\mathbb{T})}^{2}+\sum_{i, j=1}^{2}\left\|\mathbf{n}_{x_{i} x_{j}}(t)\right\|_{L_{2}(\mathbb{T})}^{2}+\|\nabla \mathbf{u}\|_{L_{2}(\mathbb{T})}^{2}\right)\right. \\
\left.+\operatorname{esssup}\|\nabla \mathbf{n}(t)\|\left(\|\nabla \boldsymbol{\nu}(t)\|_{L_{2}(\mathbb{T})}\|\Delta \mathbf{n}(t)\|_{L_{2}(\mathbb{T})}\right)\right) d t .
\end{gathered}
$$

Since $\sum_{i j}\left\|\mathbf{u}_{x_{i} x_{j}}\right\|_{L_{2}(\mathbb{T})}^{2}=\|\Delta \mathbf{u}\|_{L_{2}(\mathbb{T})}^{2}($ see $[24$, III, $\S 8])$, we have estimated $\mathbf{u}$ in the $L_{2}\left((0, T) ; W_{2}^{2}(\mathbb{T})\right)$ norm.

We need to estimate esssup $\|\nabla \mathbf{u}(t)\|$ and esssup $\|\nabla \mathbf{n}(t)\|$ in terms of higher derivatives. Unfortunately, the $W_{2}^{2}$-norm is not enough, so we repeat the previous procedure for $(\boldsymbol{\omega}, \boldsymbol{\zeta}, \boldsymbol{\psi})=\left(\Delta^{2} \mathbf{u}, \Delta^{2} \boldsymbol{\nu},-K \Delta^{3} \mathbf{n}\right)$. We use below the identity $\|\nabla(\Delta \mathbf{u})\|^{2}:=\Delta \mathbf{u}_{x_{k}} \cdot \Delta \mathbf{u}_{x_{k}}$. From (13)-(15), we have

$$
\begin{aligned}
\frac{1}{2} \int_{\mathbb{T}} & \left.\left(J\|\Delta \boldsymbol{\nu}\|^{2}+\|\Delta \mathbf{u}\|^{2}+K\|\nabla(\Delta \mathbf{n})\|^{2}\right) d x_{1} d x_{2}\right|_{0} ^{t}+\int_{Q_{t}} \mu\|\nabla(\Delta \mathbf{u})\|^{2} d x_{1} d x_{2} d t \\
\leq & -\int_{Q_{t}}\left(u_{x_{k}}^{j}\left(2 J \boldsymbol{\nu}_{x_{j} x_{k}} \cdot \Delta \boldsymbol{\nu}+2 \mathbf{u}_{x_{j} x_{k}} \cdot \Delta \mathbf{u}+K \Delta \mathbf{n}_{x_{j}} \cdot \Delta \mathbf{n}_{x_{k}}+2 K \mathbf{n}_{x_{i} x_{j} x_{k}} \cdot \Delta \mathbf{n}_{x_{i}}\right)\right. \\
& +\Delta u^{j}\left(J \boldsymbol{\nu}_{x_{j}} \cdot \Delta \boldsymbol{\nu}+\mathbf{u}_{x_{j}} \cdot \Delta \mathbf{u}+K \mathbf{n}_{x_{j} x_{k}} \cdot \Delta \mathbf{n}_{x_{k}}\right)+K u_{x_{k} x_{i}}^{j} \mathbf{n}_{x_{j} x_{k}} \cdot \Delta \mathbf{n}_{x_{i}} \\
& +K \Delta u_{x_{k}}^{j} \mathbf{n}_{x_{j}} \cdot \Delta \mathbf{n}_{x_{k}}-K\left(\Delta \mathbf{n}_{x_{k}} \cdot \mathbf{n}_{x_{j}}+\mathbf{n}_{x_{j} x_{k}} \cdot \Delta \mathbf{n}\right) \Delta u_{x_{k}}^{j} \\
& +K\left(\Delta^{2} \mathbf{n} \times \mathbf{n}+2\left(\Delta \mathbf{n}_{x_{k}} \times \mathbf{n}_{x_{k}}\right)\right) \cdot \Delta \boldsymbol{\nu}+K(\Delta \boldsymbol{\nu} \times \mathbf{n}) \cdot \Delta^{2} \mathbf{n} \\
& \left.-K\left(2 \boldsymbol{\nu}_{x_{j} x_{k}} \times \mathbf{n}_{x_{j}}+2 \boldsymbol{\nu}_{x_{j}} \times \mathbf{n}_{x_{j} x_{k}}+\boldsymbol{\nu}_{x_{k}} \times \Delta \mathbf{n}+\boldsymbol{\nu} \times \Delta \mathbf{n}_{x_{k}}\right) \cdot \Delta \mathbf{n}_{x_{k}}\right) d x_{1} d x_{2} d t \\
\leq & C \int_{0}^{T}\left(\operatorname{esssup}\|\nabla \mathbf{u}(t)\|\left(\|\boldsymbol{\nu}(t)\|_{W_{2}^{2}(\mathbb{T})}^{2}+\|\mathbf{u}(t)\|_{W_{2}^{2}(\mathbb{T})}^{2}+\|\mathbf{n}(t)\|_{W_{2}^{3}(\mathbb{T})}^{2}\right)\right. \\
& +\left(\|\mathbf{u}\|_{W_{4}^{2}(\mathbb{T})}+\|\boldsymbol{\nu}\|_{W_{4}^{1}(\mathbb{T})}\right)\left(\|\boldsymbol{\nu}(t)\|_{W_{4}^{1}(\mathbb{T})}+\|\mathbf{u}(t)\|_{W_{4}^{1}(\mathbb{T})}\right. \\
& \left.+\|\mathbf{n}(t)\|_{W_{4}^{2}(\mathbb{T})}\right)\left(\|\boldsymbol{\nu}(t)\|_{W_{2}^{2}(\mathbb{T})}+\|\mathbf{u}(t)\|_{W_{2}^{2}(\mathbb{T})}+\|\mathbf{n}(t)\|_{W_{2}^{3}(\mathbb{T})}\right) \\
& +\|\mathbf{u}\|_{W_{2}^{3}(\mathbb{T})}\|\mathbf{n}(t)\|_{W_{4}^{2}(\mathbb{T})}^{2}+\operatorname{esssup}\|\nabla \mathbf{n}(t)\|\left(\|\boldsymbol{\nu}(t)\|_{W_{2}^{2}(\mathbb{T})}\|\mathbf{n}(t)\|_{W_{2}^{3}(\mathbb{T})}\right) \\
& \left.+\operatorname{esssup}\|\boldsymbol{\nu}(t)\|\|\mathbf{n}(t)\|_{W_{2}^{3}(\mathbb{T})}^{2}\right) d t .
\end{aligned}
$$


Since for any periodic $f \in W_{2}^{1}\left([0,1]^{2}\right)$, we have

$$
\|f\|_{L_{p}} \leq C\left(\|\nabla f\|_{L_{2}}+\|f\|_{L_{2}}\right) \text { for any } p<\infty,
$$

and any periodic $g \in W_{p}^{1}\left([0,1]^{2}\right)$,

$$
\max \|g\| \leq C\left(\|\nabla g\|_{L_{p}}+\|g\|_{L_{2}}\right) \quad \text { for } p>2,
$$

we conclude from Lemma 2.1, (22), (21), and (23) that

$$
\mathscr{I}(t) \leq \mathscr{I}(0)+C_{4} \int_{0}^{t} \mathscr{I}(t)^{2} d t, \quad \text { as } t<T_{1},
$$

where

$$
\mathscr{I}(t):=\|\mathbf{u}(t)\|_{W_{2}^{2}(\mathbb{T})}^{2}+J\|\boldsymbol{\nu}(t)\|_{W_{2}^{2}(\mathbb{T})}^{2}+K\|\mathbf{n}(t)\|_{W_{2}^{3}(\mathbb{T})}^{2}+1,
$$

and the time $T_{1}$ depends only on initial data, and the positive constants $J, K, \mu$. Indeed, due to the Cauchy inequality and (24), (25)

$$
\int_{0}^{T} \operatorname{esssup}\|\nabla \mathbf{u}\|(\mathscr{I}(t)-1) d t \leq \varepsilon \int_{0}^{T}\|\nabla(\Delta \mathbf{u}(t))\|_{L_{2}(\mathbb{T})}^{2} d t+C_{\varepsilon} \int_{0}^{T} \mathscr{I}^{2}(t) d t
$$

for any $\varepsilon>0$ and some $C_{\varepsilon}>0$. The same inequality holds for the terms containing $\|\mathbf{u}\|_{W_{4}^{2}(\mathbb{T})}$ or $\|\mathbf{u}\|_{W_{2}^{3}(\mathbb{T})}$. The rest of the terms can be estimated with $C \int_{0}^{t} \mathscr{I}^{\frac{3}{2}} d t \leq C \int_{0}^{t} \mathscr{I}^{2} d t$. If $\varepsilon$ is sufficiently small, we get (26).

Next, we need the following simple lemma, a kind of Gronwall-Bellman inequality.

Lemma 2.2. Let $Y$ be a measurable function on $\mathbb{R}$. Suppose that for almost all $t$ we have

$$
0 \leq Y(t) \leq Y(0)+k \int_{0}^{t} Y(s)^{2} d s .
$$

Then

$$
Y(t) \leq \frac{Y(0)}{1-k t Y(0)}
$$

Proof. Define the function $X(t)$ by the integral equation

$$
\begin{aligned}
& X(t)=Y(0)+\varepsilon+k \int_{0}^{t} X(s)^{2} d s \Longleftrightarrow X^{\prime}=k X^{2}, \quad X(0)=Y(0)+\varepsilon, \\
& \text { i.e., } \quad X(t)=\frac{Y(0)+\varepsilon}{1-k t(Y(0)+\varepsilon)} .
\end{aligned}
$$

The difference $W=Y-X$ satisfies the inequality

$$
W(t) \leq-\varepsilon+k \int_{0}^{t}(X(s)+Y(s)) W(s) d s .
$$

Since $Y$ and $X$ are measurable, the function

$$
f(t)=-\varepsilon+\int_{0}^{t}(X(s)+Y(s)) W(s) d s
$$

is continuous. Suppose $t_{0}$ is the least zero of $f$. Since $X+Y>0$, the condition $f\left(t_{0}\right)=0$ means that $W$ is non-negative on a positive measure subset of $\left(0, t_{0}\right)$. But this is in contradiction with $f(t)<0$ as $t<t_{0}$; consequently $f(t)<0$ for all $t>0$.

Letting $\varepsilon \rightarrow 0$, yields the statement in the lemma. 
We continue the proof of the theorem. From (26), we conclude that for any $t<\min \left\{T_{1},\left(C_{4} \mathscr{I}(0)\right)^{-1} / 2\right\}$ $=: T_{2}$ we have

$$
\mathscr{I}(t) \leq C_{5},
$$

where $C_{5}$ depends only on $K, \mu, J, \mathscr{I}(0)$. This proves the first set of three inequalities in the statement of the theorem.

Using Lemma 2.2 we can now estimate the time-derivative $\mathbf{u}_{t}$. Set $\boldsymbol{\omega}=\mathbf{u}_{t}$ in (13) and rewrite the resulting identity as

$$
\begin{aligned}
& \int_{Q_{T_{2}}}\left\|\mathbf{u}_{t}\right\|^{2} d x_{1} d x_{2} d t+\left.\frac{1}{2} \mu \int_{\mathbb{T}}\|\nabla \mathbf{u}\|^{2} d x_{1} d x_{2}\right|_{0} ^{t} \\
& =\int_{Q_{T_{2}}}\left(-u^{j} \mathbf{u}_{x_{j}} \cdot \mathbf{u}_{t}+\Delta \mathbf{n} \cdot \mathbf{n}_{x_{j}} u_{t}^{j}\right) d x_{1} d x_{2} d t .
\end{aligned}
$$

Since $u^{j} \mathbf{u}_{x_{j}}$ and $\Delta \mathbf{n} \cdot \mathbf{n}_{x_{j}}$ are uniformly bounded in $L_{2}\left(Q_{t}\right)$ [which follows from (27) and standard embedding theorems], we conclude

$$
\int_{Q_{t}}\left\|\mathbf{u}_{t}\right\|^{2}+\left.\mu \int_{Q_{t}}\|\nabla \mathbf{u}\|^{2} d x_{1} d x_{2}\right|_{0} ^{t} \leq\left\|u^{j} \mathbf{u}_{x_{j}}\right\|_{L_{2}\left(Q_{t}\right)}^{2}+\|\Delta \mathbf{n} \cdot \nabla \mathbf{n}\|_{L_{2}\left(Q_{t}\right)}^{2} \leq C_{6} .
$$

The same type of inequalities can be obtained in a similar fashion for $\boldsymbol{\nu}_{t}$ and $\nabla \mathbf{n}_{t}$. This proves the second set of inequalities in the statement of the theorem.

\subsection{Convergence of the Approximations}

In this subsection we finish the proof of Theorem 2.1.

Theorem 2.2 provides the existence of measurable functions $\mathbf{u}, \boldsymbol{\nu}, \mathbf{n}$ and a subsequence of $\left(\mathbf{u}_{k}, \boldsymbol{\nu}_{k}, \mathbf{n}_{k}\right)$ such that

$$
\begin{aligned}
& \mathbf{u}_{k} \rightarrow \mathbf{u} \quad \text { weakly in } L_{2}\left((0, T) ; S o l_{2}^{2}(\mathbb{T})\right), \quad \mathbf{u}_{k, t} \rightarrow \mathbf{u}_{t} \quad \text { weakly in } \operatorname{Sol}_{2}\left(Q_{T}\right), \\
& \boldsymbol{\nu}_{k} \stackrel{*}{\rightarrow} \boldsymbol{\nu} \quad{ }^{*} \text {-weakly in } L_{\infty}\left((0, T) ; W_{2}^{2}(\mathbb{T})\right), \\
& \mathbf{n}_{k} \stackrel{*}{\rightarrow} \mathbf{n} \quad{ }^{*} \text {-weakly in } L_{\infty}\left((0, T) ; W_{2}^{3}(\mathbb{T})\right), \\
& \mathbf{u}_{k, t} \rightarrow \mathbf{u}_{t}, \quad \boldsymbol{\nu}_{k, t} \rightarrow \boldsymbol{\nu}_{t}, \quad \nabla \mathbf{n}_{k, t} \rightarrow \nabla \mathbf{n}_{t} \quad \text { weakly in } L_{2}\left(Q_{T}\right) .
\end{aligned}
$$

Moreover, due to standard embedding theorems,

$$
\mathbf{u}_{k} \rightarrow \mathbf{u} \quad \text { strongly in } \operatorname{Sol}_{2}\left(Q_{T}\right), \quad \boldsymbol{\nu}_{k} \rightarrow \boldsymbol{\nu} \text { strongly in } L_{2}\left(Q_{T}\right)
$$

and

$$
\nabla \mathbf{n}_{k} \rightarrow \nabla \mathbf{n} \quad \text { strongly in } L_{2}\left(Q_{T}\right) .
$$

Fix $\boldsymbol{\omega} \in \cup_{k} C^{1}\left(0, T ; E^{k}\right), \boldsymbol{\zeta}(\boldsymbol{t}), \boldsymbol{\psi}(\boldsymbol{t}) \in \cup_{k} C^{1}\left(0, T ; F^{k}\right)$ and integrate (13)-(15) over $(0, T)$. Passing to the limits as $k \rightarrow \infty$ we have

$$
\left\{\begin{array}{l}
\int_{Q_{T}}\left(\dot{\mathbf{u}}-\mu \Delta \mathbf{u}-K \Delta n^{i} \nabla n^{i}\right) \cdot \boldsymbol{\omega} d x_{1} d x_{2} d t=0 \\
\int_{Q_{T}}\left(\frac{J}{K} \dot{\boldsymbol{\nu}} \cdot \boldsymbol{\zeta}+(\Delta \mathbf{n} \times \mathbf{n}) \cdot \boldsymbol{\zeta}\right) d x_{1} d x_{2} d t=0 \\
\int_{Q_{T}}(\dot{\mathbf{n}} \cdot \boldsymbol{\psi}-(\boldsymbol{\nu} \times \mathbf{n}) \cdot \boldsymbol{\psi}) d x_{1} d x_{2} d t=0
\end{array}\right.
$$

Since $\cup_{k} C^{1}\left((0, T) ; E^{k}\right)$ is dense in $L_{2}\left((0, T) ; S \operatorname{Sol}_{2}(\mathbb{T})\right)$ and $\cup_{k} C^{1}\left((0, T) ; F^{k}\right)$ is dense in $L_{2}\left(Q_{T}\right)$, these equations imply $(9)-(11)$.

If $\nabla p^{\prime}$ is the Hodge projection of $\dot{\mathbf{u}}-\mu \Delta \mathbf{u}-\Delta \mathbf{n} \nabla \mathbf{n} \in L_{2}\left(Q_{T}\right)$ on the orthogonal complement of $L_{2}\left((0, T) ; \operatorname{Sol}_{2}(\mathbb{T})\right)$, then $\nabla p^{\prime} \in L_{2}\left(Q_{T}\right)$.

Finally, we check the initial conditions (12). 
Fix $\phi \in \operatorname{Sol}_{2}(\mathbb{T})$ and consider the family of functions $f_{k}(t)=\left(\mathbf{u}_{k}(t), \phi\right)_{L_{2}(\mathbb{T})}$. Since $f_{k}^{\prime}(t)=$ $\left(\mathbf{u}_{k, t}(t), \boldsymbol{\phi}\right)_{L_{2}(\mathbb{T})} \rightarrow\left(\mathbf{u}_{t}, \boldsymbol{\phi}\right)_{L_{2}(\mathbb{T})}$ weakly in $L_{2}(0, T)$, it follows that $f_{k}$ tends to $(\mathbf{u}, \boldsymbol{\phi})_{L_{2}(\mathbb{T})}$ in $C(0, T)$. Thus, $\mathbf{u}(t, \cdot)$ tends to $\lim _{k} \mathbf{u}_{0 k}=\mathbf{u}_{0}$ weakly in $L_{2}(\mathbb{T})$. Also

$$
\int_{\mathbb{T}}(\mathbf{u}(t, \mathbf{x})-\mathbf{u}(0, \mathbf{x}))^{2} d x_{1} d x_{2}=\int_{\mathbb{T}}\left(\int_{0}^{t} \mathbf{u}_{t} d t\right)^{2} d x_{1} d x_{2} \leq t \int_{\mathbb{T}} \int_{0}^{t} \mathbf{u}_{t}^{2} d t d x_{1} d x_{2} .
$$

Since $\mathbf{u}_{t} \in L_{2}\left(Q_{t}\right)$, the function $u(t, x)$ is continuous in $L_{2}(\mathbb{T})$-norm with respect to $t$. Consequently, $\mathbf{u}_{0}$ is both a weak and a strong limit.

The weak limits of the other variables to their respective initial conditions are checked in the same way. This proves Theorem 2.1.

\subsection{Uniqueness}

Theorem 2.3. Suppose that $\left(\mathbf{u}_{1}, \boldsymbol{\nu}_{1}, \mathbf{n}_{1}, p_{1}^{\prime}\right)$ and $\left(\mathbf{u}_{2}, \boldsymbol{\nu}_{2}, \mathbf{n}_{2}, p_{2}^{\prime}\right)$ are solutions of the problem (9)-(12) in the domain $Q_{T}$. Then, for some $0<T_{0} \leq T$

$$
\left(\mathbf{u}_{2}, \boldsymbol{\nu}_{2}, \mathbf{n}_{2}, \nabla p_{2}^{\prime}\right)=\left(\mathbf{u}_{1}, \boldsymbol{\nu}_{1}, \mathbf{n}_{1}, \nabla p_{1}^{\prime}\right)
$$

almost everywhere in $Q_{T_{0}}$.

Proof. First of all, every solution of the problem satisfies identities (28) for all $\boldsymbol{\omega} \in W_{2}^{1}\left(Q_{T}\right), \boldsymbol{\zeta} \in W_{2}^{1}\left(Q_{t}\right)$, $\psi \in L_{2}\left(0, T ; W_{2}^{2}\right)$.

Denote $\mathbf{w}=\mathbf{u}_{1}-\mathbf{u}_{2}, \mathbf{f}=\boldsymbol{\nu}_{\mathbf{1}}-\boldsymbol{\nu}_{\mathbf{2}}, \mathbf{g}=\mathbf{n}_{1}-\mathbf{n}_{2}$ and set $(\boldsymbol{\omega}, \boldsymbol{\zeta}, \boldsymbol{\psi})=(\mathbf{w}, \mathbf{f},-K \Delta \mathbf{g})$ in (28). With this substitution, for any $\tau<T$, the identities (28) give

$$
\begin{aligned}
& \int_{Q_{\tau}}\left(\mathbf{w}_{t} \cdot \mathbf{w}+u_{2}^{i} \mathbf{w}_{x_{i}} \cdot \mathbf{w}+\mathrm{w}^{i} \mathbf{u}_{1, x_{i}} \cdot \mathbf{w}+\mu\|\nabla \mathbf{w}\|^{2}\right. \\
& \left.\quad+K \Delta \mathbf{n}_{1} \cdot \mathbf{g}_{x_{j}} \mathrm{w}^{i}+K \Delta \mathbf{g} \cdot \mathbf{n}_{2, x_{j}} \mathrm{w}^{j}\right) d x_{1} d x_{2} d t=0 \\
& \int_{Q_{\tau}}\left(J\left(\mathbf{f}_{t} \cdot \mathbf{f}+u_{2}^{i} \mathbf{f}_{x_{i}} \cdot \mathbf{f}+\mathrm{w}^{i} \boldsymbol{\nu}_{1, x_{i}} \cdot \mathbf{f}\right)\right. \\
& \left.\quad+K\left(\Delta \mathbf{g} \times \mathbf{n}_{1}+\Delta \mathbf{n}_{2} \times \mathbf{g}\right) \cdot \mathbf{f}\right) d x_{1} d x_{2} d t=0 \\
& \quad-K \int_{Q_{\tau}}\left(\mathbf{g}_{t} \cdot \Delta \mathbf{g}+u_{1}{ }^{i} \mathbf{g}_{x_{i}} \cdot \Delta \mathbf{g}+\mathrm{w}^{i} \mathbf{n}_{2, x_{i}} \cdot \Delta \mathbf{g}\right. \\
& \left.\quad-\left(\boldsymbol{\nu}_{\mathbf{1}} \times \mathbf{g}+\mathbf{f} \times \mathbf{n}_{2}\right) \cdot \Delta \mathbf{g}\right) d x_{1} d x_{2} d t=0
\end{aligned}
$$

Next, we rewrite these identities as

$$
\begin{aligned}
& \int_{\mathbb{T}} \frac{1}{2}\|\mathbf{w}(\tau)\|^{2} d^{2} x+\int_{Q_{\tau}} \mu\|\nabla \mathbf{w}\|^{2} d^{2} x d t \\
& =\int_{Q_{\tau}}\left(-\mathrm{w}^{i} \mathbf{u}_{1, x_{i}} \cdot \mathbf{w}-K\left(\Delta \mathbf{n}_{1} \cdot \mathbf{g}_{x_{j}} \mathrm{w}^{i}+\Delta \mathbf{g} \cdot \mathbf{n}_{2, x_{j}} \mathrm{w}^{j}\right) d x_{1} d x_{2} d t,\right. \\
& \frac{J}{2} \int_{\mathbb{T}}\|\mathbf{f}(\tau)\|^{2} d x_{1} d x_{2} \\
& =-\int_{Q_{\tau}}\left(J_{\mathrm{W}}{ }^{i} \boldsymbol{\nu}_{1, x_{i}} \cdot \mathbf{f}+K\left(\Delta \mathbf{g} \times \mathbf{n}_{2}+\Delta \mathbf{n}_{1} \times \mathbf{g}\right) \cdot \mathbf{f}\right) d x_{1} d x_{2} d t, \\
& \frac{K}{2} \int_{\mathbb{T}}\|\nabla \mathbf{g}(\tau)\|^{2} d x_{1} d x_{2} \\
& =K \int_{Q_{\tau}}\left(\mathrm{w}^{i} \mathbf{n}_{2, x_{i}} \cdot \Delta \mathbf{g}-u_{1, x_{k}}^{i} \mathbf{g}_{x_{i}} \cdot \mathbf{g}_{x_{k}}+\left(\boldsymbol{\nu}_{1, x_{k}} \times \mathbf{g}\right) \cdot \mathbf{g}_{x_{k}}\right. \\
& \left.-\left(\mathbf{f} \times \mathbf{n}_{2}\right) \cdot \Delta \mathbf{g}\right) d x_{1} d x_{2} d t .
\end{aligned}
$$


Adding the identities (32)-(34), we get

$$
\begin{aligned}
& \frac{1}{2} \int_{\mathbb{T}}\left(\|\mathbf{w}(\tau)\|^{2}+J\|\mathbf{f}(\tau)\|^{2}+K\|\nabla \mathbf{g}(\tau)\|^{2}\right) d x_{1} d x_{2}+\mu \int_{Q_{\tau}}\|\nabla \mathbf{w}(t)\|^{2} d x_{1} d x_{2} d t \\
& =-\int_{Q_{\tau}}\left(\mathrm{w}^{j} u_{1, x_{j}}^{i} \mathrm{w}^{i}+K \Delta \mathbf{n}_{1} \cdot \mathbf{g}_{x_{j}} \mathrm{w}^{i}+J \mathrm{w}^{i} \boldsymbol{\nu}_{1, x_{i}} \cdot \mathbf{f}+K\left(\Delta \mathbf{n}_{1} \times \mathbf{g}\right) \cdot \mathbf{f}\right. \\
& \left.\quad+K u_{1, x_{k}}^{i} \mathbf{g}_{x_{i}} \cdot \mathbf{g}_{x_{k}}-K\left(\boldsymbol{\nu}_{1, x_{k}} \times \mathbf{g}\right) \cdot \mathbf{g}_{x_{k}}\right) d x_{1} d x_{2} d t .
\end{aligned}
$$

Due to the embedding theorems and the Hölder inequalities we get

$$
\begin{aligned}
& \frac{1}{2} \int_{\mathbb{T}}\left(\|\mathbf{w}(\tau)\|^{2}+J\|\mathbf{f}(\tau)\|^{2}+K\|\nabla \mathbf{g}(\tau)\|^{2}\right) d x_{1} d x_{2}+\mu \int_{Q_{\tau}}\|\nabla \mathbf{w}(t)\|^{2} d x_{1} d x_{2} d t \\
& \leq C \int_{0}^{\tau}\left[\left(\|\mathbf{f}\|_{L_{2}(\mathbb{T})}\left\|\nabla \nu_{1}\right\|_{L_{q}(\mathbb{T})}+\|\nabla \mathbf{g}\|_{L_{2}(\mathbb{T})}\left\|\Delta \mathbf{n}_{1}\right\|_{L_{q}(\mathbb{T})}\right)\|\mathbf{w}\|_{L_{p}(\mathbb{T})}\right. \\
& \quad+\left(\|\mathbf{g}\|_{L_{2}(\mathbb{T})}\left\|\nabla \boldsymbol{\nu}_{1}\right\|_{L_{q}(\mathbb{T})}+\|\nabla \mathbf{f}\|_{L_{2}(\mathbb{T})}\left\|\Delta \mathbf{n}_{1}\right\|_{L_{q}(\mathbb{T})}\right)\|\mathbf{g}\|_{L_{p}(\mathbb{T})} \\
&\left.\quad+\left\|\nabla \mathbf{u}_{1}\right\|_{L_{\infty}(\mathbb{T})}\left(\|\mathbf{w}\|_{L_{2}(\mathbb{T})}^{2}+\|\nabla \mathbf{g}\|_{L_{2}(\mathbb{T})}^{2}\right)\right] d t \\
& \leq C(\delta) \tau^{\frac{1}{2}}\left[\underset{t}{\operatorname{esssup}}\left(\left\|\Delta \mathbf{n}_{1}(t)\right\|_{L_{q}(\mathbb{T})}+\left\|\nabla \boldsymbol{\nu}_{1}(t)\right\|_{L_{q}(\mathbb{T})}\right)\right. \\
&\left.\quad+\left\|\nabla \mathbf{u}_{1}(t)\right\|_{L_{2}\left((0, T) ; L_{\infty}(\mathbb{T})\right)}\right]\left(\underset { t } { \operatorname { e s s s u p } } \left(\|\mathbf{w}(t)\|_{L_{2}(\mathbb{T})}^{2}\right.\right. \\
&\left.\left.\quad+\|\mathbf{f}(t)\|_{L_{2}(\mathbb{T})}^{2}+\|\nabla \mathbf{g}(t)\|_{L_{2}(\mathbb{T})}^{2}\right)+\|\nabla \mathbf{w}(t)\|_{L_{2}\left(Q_{\tau}\right)}^{2}\right)
\end{aligned}
$$

for some $q>2, p^{-1}=1 / 2-q^{-1}$.

Taking the $\tau$-esssup of the left hand side and comparing it to the second factor on the right hand side, shows that for $\tau$ sufficiently small we have

$$
(\mathbf{w}, \mathbf{f}, \nabla \mathbf{g})=(0,0,0) \text {. }
$$

Consequently, $\left(\mathbf{u}_{1}, \boldsymbol{\nu}_{\mathbf{1}}\right)=\left(\mathbf{u}_{2}, \boldsymbol{\nu}_{\mathbf{2}}\right)$. However, $\dot{\mathbf{g}}=-\mathrm{w}^{i} \mathbf{n}_{2, x_{i}}+\boldsymbol{\nu}_{\mathbf{1}} \times \mathbf{g}+\mathbf{f} \times \mathbf{n}_{2}$. Since $\mathbf{f}=0$ and $\mathbf{w}=0$, we have $\frac{d}{d t} \mathbf{g}(t)=\boldsymbol{\nu}_{\mathbf{1}}(t) \times \mathbf{g}(t)$ with initial condition $\mathbf{g}(0)=0$. This implies that $\mathbf{g}=0$, i.e., $\mathbf{n}_{1}=\mathbf{n}_{2}$. Since $\nabla p_{i}$ is the projection of $\dot{\mathbf{u}}-\mu \Delta \mathbf{u}-\Delta \mathbf{n} \nabla \mathbf{n}$, we have $\nabla p_{1}=\nabla p_{2}$.

Theorem 2.3 is proved.

\subsection{Liquid Crystal in the Presence of External Forces}

Theorem 2.1 can be easily extended if $\mathbf{F}, \mathbf{G} \neq 0$.

Theorem 2.4. Suppose $\mathbf{u}_{0} \in \operatorname{Sol}_{2}^{2}(\mathbb{T}), \boldsymbol{\nu}_{0} \in W_{2}^{2}(\mathbb{T}), \mathbf{n}_{0} \in W_{2}^{3}(\mathbb{T})$ and $\mathbf{F} \in L_{2}\left((0, T) ; W_{2}^{1}(\mathbb{T})\right)$, G $\in$ $L_{1}\left((0, T) ; W_{2}^{2}(\mathbb{T})\right) ; F^{3}=0$.

Then there exists some $0<T_{0}<T$ such that the solution (as in Definition 2.1) of problem (9)-(11) exists and is unique in $Q_{T_{0}}$.

\section{Liquid Crystal Flow in Bounded Domains}

Let $\Omega$ be a bounded domain in $\mathbb{R}^{2}$ and consider nematic liquid crystal flow in the cylinder $\Omega \times \mathbb{R}$ which does not depend on the third coordinate.

Since all functions in the Ericksen-Leslie system depend only on the points $\left(x_{1}, x_{2}\right) \in \Omega$, we are studying Eqs. (9)-(11) in domain $(0, T) \times \Omega$ with initial conditions (12) and additional boundary conditions

$$
\left.\mathbf{u}\right|_{\partial \Omega}=0, \quad \mathbf{n}-\left.\mathbf{n}_{1}\right|_{\partial \Omega}=0,\left.\quad \boldsymbol{\nu}\right|_{\partial \Omega}=0 \quad \text { for any } t>0
$$

where $\mathbf{n}_{1}$ is a given vector field on $\Omega \times \mathbb{R}$. 
Condition $\left.\mathbf{u}\right|_{\partial \Omega}=0$ means that the domain has impenetrable boundary and that the fluid moves without slipping; $\mathbf{n}-\left.\mathbf{n}_{\mathbf{1}}\right|_{\partial \Omega}=0$ describes the director position at the boundary. The third condition comes from the original Ericksen-Leslie system and means that $\dot{\mathbf{n}}=0$ at the boundary.

\subsection{The Definition of the Solution and the Existence Theorem}

We begin by introducing some notations.

In this section we let $Q_{T}:=(0, T) \times \Omega$,

$$
\begin{aligned}
& \left.\stackrel{\circ}{\operatorname{Sol}}(\Omega):=\left\{\mathbf{v}: \Omega \rightarrow \mathbb{R}^{3} \text { such as } \mathbf{v} \in C_{0}^{\infty} \Omega\right), \operatorname{div} \mathbf{v}=0\right\} \\
& \stackrel{\circ}{\operatorname{Sol}}\left(Q_{T}\right):=\left\{\mathbf{v} \in C^{\infty}\left(Q_{T}\right): \forall t \mathbf{v}(t, \cdot) \in \operatorname{Sol}(\Omega)\right\} \\
& \operatorname{Sol}_{2}^{m}(\Omega) \text { is the closure of } \operatorname{Sol}(\Omega) \text { in the norm } W_{2}^{m}(\Omega)
\end{aligned}
$$

$\stackrel{\circ}{W_{2}^{m}}(\Omega)$ is the subspace of $W_{2}^{m}(\Omega)$ with zero trace (see, for instance $[25,26]$ ).

The definition of a solution of the Ericksen-Leslie equations is quite similar to the one in Definition 2.1, with some changes because of the boundary.

Definition 3.1. The quadruple $\left(\mathbf{u}, \boldsymbol{\nu}, \mathbf{n}, \nabla p^{\prime}\right)$ is a strong solution of problem (9)-(12), (35) in domain $Q_{T}$ if

- $\mathbf{u}$ is a vector field in $L_{2}\left((0, T) ; S o l_{2}^{1}(\Omega)\right) \cap L_{2}\left((0, T) ; W_{2}^{2}(\Omega)\right), \mathbf{u}_{t} \in L_{2}\left(Q_{T}\right)$;

- $\boldsymbol{\nu}$ is a vector field in $L_{\infty}\left((0, T) ; W_{2}^{1}(\Omega)\right), \boldsymbol{\nu}_{t} \in L_{\infty}\left((0, T) ; L_{2}(\Omega)\right)$;

- $\mathbf{n}-\mathbf{n}_{1}$ is a vector field in $L_{\infty}\left((0, T) ; \stackrel{\circ}{W_{2}^{1}}(\Omega)\right) \cap L_{2}\left((0, T) ; W_{2}^{2}(\Omega)\right)$, where $\mathbf{n}_{1}$ is a given constant vector field in $(x, t), \mathbf{n}_{t} \in L_{\infty}\left((0, T) ; W_{2}^{1}(\Omega)\right)$;

- $\nabla p^{\prime} \in L_{2}\left(Q_{T}\right)$;

- $\mathbf{u}, \mathbf{n}, \boldsymbol{\nu}$ satisfy initial conditions $(12)$, i.e., $(\mathbf{u}, \mathbf{n}, \boldsymbol{\nu}) \rightarrow\left(\mathbf{u}_{0}, \mathbf{n}_{0}, \boldsymbol{\nu}_{0}\right)$ in $L_{2}(\Omega)$ as $t \rightarrow 0$;

- Equations (9)-(11) hold almost everywhere.

In this section we suppose the third component of the director to be equal to zero. Then we have

$$
\mathbf{n}=(\cos \theta, \sin \theta, 0), \quad \boldsymbol{\nu}=(0,0, \nu),
$$

where $\theta$ is a new unknown function. The Ericksen-Leslie system becomes

$$
\begin{aligned}
\dot{\mathbf{u}}-\mu \Delta \mathbf{u} & =-\nabla\left(p^{\prime}+\frac{K}{2}\|\nabla \theta\|^{2}\right)-K \Delta \theta \nabla \theta, \quad \operatorname{div} \mathbf{u}=0, \\
J \dot{\nu} & =-K \Delta \theta \\
\dot{\theta} & =\nu
\end{aligned}
$$

with boundary and initial conditions

$$
\begin{aligned}
\left.\mathbf{u}\right|_{\partial \Omega} & =0, \quad \theta-\left.\theta_{1}\right|_{\partial \Omega}=0,\left.\quad \nu\right|_{\partial \Omega}=0 \quad \text { for any } t>0 \\
\mathbf{u}(0, x) & =\mathbf{u}_{0}(x), \quad \nu(0, x)=\nu_{0}(x), \quad \theta(0, x)=\theta_{0}(x)
\end{aligned}
$$

Theorem 3.1. Assume that $\Omega$ is a domain with $C^{2}$-boundary. Let $\theta_{0} \in W_{2}^{3}(\Omega), \nu_{0} \in W_{2}^{2}(\Omega)$, $\mathbf{u}_{\mathbf{0}} \in \operatorname{Sol}_{2}^{1}$ $(\Omega) \cap W_{2}^{2}(\Omega) ;\left.\Delta \mathbf{u}_{0}\right|_{\partial \Omega}=0$ and assume that for some $d>0$ we have

$$
\theta_{0}(x)=\theta_{1} \equiv \text { const }, \quad \nu_{0}(x)=0 \quad \text { if } \operatorname{dist}(x, \partial \Omega)<d .
$$

Then the solution of (36)-(40) exists for some $T>0$ and is unique. 
The proof proceeds along the same lines as that of Theorem 2.1. We point out the necessary modifications.

Instead of (36)-(38) we consider the regularized system, where equation (36) is replaced with

$$
\dot{\mathbf{u}}-\mu \Delta \mathbf{u}+\varepsilon \Delta^{2} \mathbf{u}=-\nabla P-\Delta \theta \nabla \theta
$$

with additional boundary conditions $\left.\Delta u\right|_{\partial \Omega}=0$; where $\varepsilon$ is a small parameter, $P=p^{\prime}+\frac{K}{2}\|\nabla \theta\|^{2}$.

Also, we replace $\theta$ with $\theta_{1}+\tilde{\theta}$, where $\tilde{\theta}$ is a new unknown function.

As in the proof of Theorem 2.1, select two sequences of subspaces $E^{1} \subset E^{2} \subset \cdots$ and $F^{1} \subset F^{2} \subset \cdots$ such that $\cup_{k} E^{k}$ is dense in $\operatorname{Sol}_{2}^{1}(\Omega) \cap W_{2}^{2}(\Omega)$ and $\cup_{k} F^{k}$ is dense in $W_{2}^{2}(\Omega) \cap \stackrel{\circ}{W_{2}^{1}}(\Omega)$. It is still useful to choose $E^{k}$ and $F^{k}$ to be the linear span of the first $k$ eigenfunctions of the Laplacian in the corresponding spaces.

Define the finite-dimensional solution $\left(\mathbf{u}_{k}, \nu_{k}, \tilde{\theta}_{k}\right) \subset C^{0}\left((0, T) ; E^{k} \times F^{k} \times F^{k}\right)$ by the system of ordinary differential equations

$$
\begin{aligned}
\left(\mathbf{u}_{k, t}, \boldsymbol{\omega}\right)= & -\left(u_{k}^{l} \mathbf{u}_{k, x_{l}}, \boldsymbol{\omega}\right)+\left(\mu \Delta \mathbf{u}_{k}-\varepsilon \Delta^{2} \mathbf{u}_{k}, \boldsymbol{\omega}\right) \\
& -K\left(\Delta\left(\tilde{\theta}_{k}+\theta_{1}\right) \cdot \nabla\left(\tilde{\theta}_{k}+\theta_{1}\right), \boldsymbol{\omega}\right), \\
J\left(\nu_{k, t}, \zeta\right)= & -\left(J u_{k}^{l} \nu_{k, x_{l}} k, \zeta\right)-K\left(\Delta\left(\tilde{\theta}_{k}+\theta_{1}\right), \zeta\right), \\
\left(\tilde{\theta}_{k, t}, \psi\right)= & -\left(u_{k}^{l}\left(\tilde{\theta}_{k}+\theta_{1}\right)_{x_{l}}, \psi\right)+\left(\nu_{k}, \psi\right), \\
\left.\left(\mathbf{u}_{k}, \nu_{k}, \theta_{k}\right)\right|_{t=0}= & \left(\mathbf{u}_{0 k}, \nu_{0 k}, \theta_{0 k}\right) .
\end{aligned}
$$

The identities (42)-(45) hold for all $\boldsymbol{\omega} \in E^{k}, \zeta, \psi \in F^{k}$. (For simplicity we use the notation $\theta_{k}=\tilde{\theta}_{k}+\theta_{1}$ ).

The solution of this finite-dimensional problem, obviously, exists for some $T>0$.

The result of Sect. 2.3 still holds.

Lemma 3.1. For all $t>0$ we have

$$
\begin{aligned}
& \left.\int_{\Omega}\left(\left\|\mathbf{u}_{\mathbf{k}}(t)\right\|^{2}+J\left\|\nu_{k}(t)\right\|^{2}+K\left\|\nabla \theta_{k}(t)\right\|^{2}\right) d x_{1} d x_{2}\right|_{0} ^{t} \\
& \quad+2 \mu \int_{0}^{t} \int_{\Omega}\left\|\nabla \mathbf{u}_{k}(s)\right\|^{2} d x_{1} d x_{2} d s+2 \varepsilon \int_{0}^{t} \int_{\Omega}\left\|\Delta \mathbf{u}_{k}(s)\right\|^{2} d x_{1} d x_{2} d s=0 .
\end{aligned}
$$

Proof. Repeat the procedure used in Lemma 2.1.

We need to check that the boundary integrals are equal to zero. Due to the condition $\left.\mathbf{u}_{k}\right|_{\partial \Omega}=0$, all terms containing $u_{k}^{i}$ vanish. The only term not containing $\mathbf{u}_{k}$ is

$$
\int_{0}^{T} \int_{\partial \Omega} \theta_{k, t} \theta_{k, x_{i}} N_{i} d S
$$

derived from (44), where $\left(N_{1}, N_{2}\right)$ is an exterior normal vector field. Since $\tilde{\theta}$ is a smooth vector field and $\theta_{1}$ does not depend on $t,\left.\theta_{t}\right|_{\partial \Omega}=0$, it follows that this term vanishes. The Lemma is proved.

As in section 2.3, Lemma 3.1 guarantees that the solution of the problem (42)-(45) exists for all $T>0$. Next, we estimate higher derivatives.

Lemma 3.2. There exists $T>0$ and $C>0$ depending on $\varepsilon$, the initial and boundary data, but not depending on $k$, such that

$$
\left\|\mathbf{u}_{k}\right\|_{L_{2}\left((0, T) ; W_{2}^{3}(\Omega)\right)},\left\|\nabla \nu_{k}\right\|_{L_{\infty}\left((0, T) ; L_{2}(\Omega)\right)},\left\|\theta_{k}\right\|_{L_{\infty}\left((0, T) ; W_{2}^{2}(\Omega)\right)} \leq C .
$$

Note that $T>0$ and $C>0$ depend on $\varepsilon$, which won't be sufficient to prove Theorem 3.1. Lemma 3.3 below gives the necessary uniform estimates. 
Proof. In this proof we use $(\mathbf{u}, \nu, \tilde{\theta})$ instead of $\left(\mathbf{u}_{k}, \nu_{k}, \tilde{\theta}_{k}\right)$.

Consider Eqs. (42)-(44) and substitute $(\boldsymbol{\omega}, \zeta, \psi)=\left(\Delta \mathbf{u}, \Delta \nu, \Delta^{2} \tilde{\theta}\right)$. First, we note that (44) could be rewritten as

$$
\begin{aligned}
\left.\frac{1}{2} \int_{\Omega}(\Delta \tilde{\theta})^{2} d x_{1} d x_{2}\right|_{0} ^{T}= & \int_{0}^{T} \int_{\partial \Omega}\left((\dot{\theta}-\nu) \Delta \theta_{x_{k}} N_{k}-\Delta \theta(\dot{\theta}-\nu)_{x_{k}} N_{k}\right) d S d t \\
& +\int_{Q_{T}}\left(-2 u_{x_{k}}^{j} \theta_{x_{j} x_{k}} \Delta \theta-\Delta u^{j} \theta_{x_{j}} \Delta \theta+\Delta \nu \Delta \theta\right) d x_{1} d x_{2} d t .
\end{aligned}
$$

The first term of the boundary integral is equal to zero, since $\dot{\theta}(t, x)=0$ and $\nu(t, x)=0$ if $x \in \partial \Omega$.

Recall that $F^{k}$ is defined to be the linear span of the first $k$ eigenfunctions $\left\{v_{m} \mid m=0, \ldots, k\right\}$ of the Laplacian, i.e.,

$$
\Delta v_{m}=\lambda v_{m},\left.\quad v_{m}\right|_{\partial \Omega}=0
$$

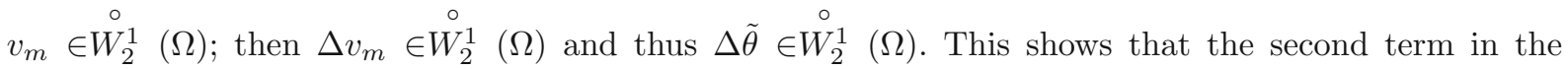
boundary integral also vanishes.

Since $\Delta \theta=\Delta \tilde{\theta}$, we have an equation similar to (20).

The analogues of (19) and (21) are obtained in the same way and, consequently, we can prove an analogue of inequality (22):

$$
\begin{aligned}
& \left.\frac{1}{2} \int_{\mathbb{T}}\left(J\|\nabla \nu\|^{2}+K \sum_{j, k=1}^{2}\left\|\theta_{x_{j} x_{k}}\right\|^{2}+\|\nabla \mathbf{u}\|^{2}\right) d x_{1} d x_{2}\right|_{0} ^{T} \\
& \quad+\mu \int_{Q_{T}}\|\Delta \mathbf{u}\|^{2} d x_{1} d x_{2} d t+\varepsilon \int_{Q_{T}}\|\nabla(\Delta \mathbf{u})\|^{2} d x_{1} d x_{2} d t \\
& \leq C_{1} \int_{0}^{T}\left(\operatorname{esssup}\|\nabla \mathbf{u}(t)\|\left(\|\nabla \nu(t)\|_{L_{2}(\Omega)}^{2}+\sum_{i, j=1}^{2}\left\|\theta_{x_{i} x_{j}}(t)\right\|_{L_{2}(\Omega)}^{2}+\|\nabla \mathbf{u}\|_{L_{2}(\Omega)}^{2}\right)\right. \\
& \left.\quad+\|\nabla \theta(t)\|_{L_{4}(\Omega)}\left(\|\Delta \mathbf{u}(t)\|_{L_{4}(\Omega)}\|\Delta \theta(t)\|_{L_{2}(\Omega)}\right)\right) d t
\end{aligned}
$$

Next, we need Lemma 8.1 of $\left[24\right.$, Chapter III] to estimate $\|\mathbf{u}\|_{W_{2}^{2}(\Omega)}$ in terms of $\|\Delta \mathbf{u}\|_{L_{2}(\Omega)}$ and $\|\mathbf{u}\|_{L_{2}(\Omega)}$, namely,

$$
\|\mathbf{u}\|_{W_{2}^{2}(\Omega)} \leq C\left(\|\Delta \mathbf{u}\|_{L_{2}(\Omega)}+\|\mathbf{u}\|_{L_{2}(\Omega)}\right)
$$

We also need inequality (11.8) from [24, Chapter III]

$$
\forall p>1 \exists C \forall v \in W_{p}^{2}(\Omega),\left.v\right|_{\partial \Omega}=0 \quad\|v\|_{W_{p}^{2}(\Omega)} \leq C\left(\|\Delta v\|_{L_{p}(\Omega)}+\|v\|_{L_{2}(\Omega)}\right)
$$

and the Sobolev embedding theorems to estimate $\|\mathbf{u}\|_{W_{p}^{2}(\Omega)}($ and, consequently, max $\|\nabla \mathbf{u}\|$ ) in terms of $\|\Delta \mathbf{u}\|_{W_{2}^{1}(\Omega)}$ and lower derivatives.

Finally, we can obtain an inequality similar to (26) for

$$
\mathscr{I}(t):=\|\mathbf{u}(t)\|_{W_{2}^{1}(\Omega)}^{2}+J\|\boldsymbol{\nu}(t)\|_{W_{2}^{1}(\Omega)}^{2}+K\|\mathbf{n}(t)\|_{W_{2}^{2}(\Omega)}^{2}+\frac{\varepsilon}{2}\|\mathbf{u}\|_{L_{2}\left((0, t) ; W_{2}^{3}(\Omega)\right)}^{2}+1,
$$

and, consequently, (27). This proves Lemma 3.2. 
Proof of Theorem 3.1. Due to Lemma 3.2, the sequences $\mathbf{u}_{k}, \nu_{k}, \theta_{k}$ are weakly-precompact in $L_{2}((0, T)$; $\left.\mathrm{Sol}_{2}^{2}(\Omega)\right), L_{\infty}\left((0, T) ; \stackrel{\circ}{W_{2}^{1}}(\Omega)\right)$, and $L_{\infty}\left((0, T) ; W_{2}^{2}(\Omega)\right)$. From the embedding theorems,

$$
\begin{aligned}
& \mathbf{u}_{k} \rightarrow \mathbf{u}^{\varepsilon} \quad \text { weakly in } L_{2}\left((0, T) ; \operatorname{Sol}_{2}^{2}(\Omega)\right), \quad \mathbf{u}_{k, t} \rightarrow \mathbf{u}_{t}^{\varepsilon} \quad \text { weakly in } \operatorname{Sol}_{2}\left(Q_{T}\right), \\
& \nu_{k} \stackrel{*}{\rightarrow} \nu^{\varepsilon} \quad \text { *-weakly in } L_{\infty}\left((0, T) ; W_{2}^{1}(\Omega)\right), \\
& \theta_{k} \stackrel{*}{\rightarrow} \theta^{\varepsilon} \quad{ }^{*} \text {-weakly in } L_{\infty}\left((0, T) ; W_{2}^{2}(\Omega)\right), \\
& \nu_{k, t} \rightarrow \nu_{t}^{\varepsilon}, \quad \nabla \theta_{k, t} \rightarrow \nabla \theta_{t}^{\varepsilon} \quad \text { weakly in } L_{2}\left(Q_{T}\right) . \\
& \mathbf{u}_{k}^{\varepsilon} \rightarrow \mathbf{u} \quad \text { strongly in } \operatorname{Sol}_{2}\left(Q_{T}\right), \quad \nu_{k} \rightarrow \nu^{\varepsilon} \quad \text { strongly in } L_{2}\left(Q_{T}\right),
\end{aligned}
$$

and

$$
\nabla \theta_{k} \rightarrow \nabla \theta^{\varepsilon} \quad \text { strongly in } L_{2}\left(Q_{T}\right)
$$

for some subsequence of $\left(\mathbf{u}_{k}, \nu_{k}, \theta_{k}\right)$.

This proves existence of solutions of the regularized problem (37), (38), (41) for any $\varepsilon>0$. To prove Theorem 3.1, it is sufficient to obtain uniform estimates for higher derivatives of $\left(\mathbf{u}^{\varepsilon}, \nu^{\varepsilon}, \theta^{\varepsilon}\right)$.

Lemma 3.3. For sufficiently small $T_{1}>0$, not depending on $\varepsilon$, there is a constant $C>0$, also not depending on $\varepsilon$, such that

$$
\begin{aligned}
& \left\|\mathbf{u}^{\varepsilon}\right\|_{L_{2}\left((0, T) ; W_{2}^{3}(\Omega)\right)},\left\|\mathbf{u}^{\varepsilon}\right\|_{L_{\infty}\left((0, T) ; W_{2}^{2}(\Omega)\right)},\left\|\nu^{\varepsilon}\right\|_{L_{\infty}\left((0, T) ; W_{2}^{2}(\Omega)\right)}, \\
& \left\|\theta^{\varepsilon}\right\|_{L_{\infty}\left((0, T) ; W_{2}^{3}(\Omega)\right)} \leq C, \\
& \left\|\mathbf{u}_{t}^{\varepsilon}\right\|_{L_{2}\left(Q_{T}\right)},\left\|\nu_{t}^{\varepsilon}\right\|_{L_{2}\left(Q_{T}\right)},\left\|\nabla \theta_{t}^{\varepsilon}\right\|_{L_{2}\left(Q_{T}\right)} \leq C .
\end{aligned}
$$

Proof. Consider, instead of (37) and (38), the equations

$$
\begin{aligned}
J \nu_{t}+\chi(x) u^{i} \nu_{x_{i}} & =-K \Delta \theta \\
\theta_{t}+\chi(x) u^{i} \theta_{x_{i}} & =\nu
\end{aligned}
$$

where $\chi(x)$ is a smooth function,

$$
\chi(x)= \begin{cases}0, & \operatorname{dist}(x, \partial \Omega)<\frac{d}{4} \\ 1, & \operatorname{dist}(x, \partial \Omega)>\frac{d}{3}\end{cases}
$$

Repeat the Galerkin procedure for the problem (39), (40), (41), (49), (50) as presented in Sect. 2. Since all nonlinear terms in (49), (50) vanish on the boundary, we can prove the analogue of Theorem 2.2.

Proposition 3.1. There exists a strong solution $(\tilde{\mathbf{u}}, \tilde{\nu}, \tilde{\theta})$ of the problem (39), (40), (41), (49), (50). The solution is unique and satisfies inequalities (47), (48), where the constants $C$ and $T$ depend on $d$ and don't depend on $\varepsilon$.

The proof is identical to that of Theorem 2.2 and the argument in Sect. 2.6, so it won't be repeated here.

Let us prove that, for some $T_{1}<T$ not depending on $\varepsilon$, the two solutions coincide, i.e.

$$
\forall t \in\left[0, T_{1}\right] \quad\left(\mathbf{u}^{\varepsilon}, \nu^{\varepsilon}, \theta^{\varepsilon}\right)(t)=(\tilde{\mathbf{u}}, \tilde{\nu}, \tilde{\theta})(t) .
$$

Define constant $T_{1}>0$ by

$$
T_{1}:=\min \left\{T, \operatorname{esssup}_{x \in \Omega ; i, j}\left\|\tilde{u}_{x_{j}}^{i}+\tilde{u}_{x_{i}}^{j}\right\|_{L_{2}(0, T)}^{2}, d(2 \underset{x, t}{\operatorname{essup}}\|\tilde{\mathbf{u}}\|+2 \max \{1, K / J\})^{-1}\right\}
$$

and let $\mathscr{K}:=\left\{t \leq T_{1} \mid\left(\mathbf{u}^{\varepsilon}, \nu^{\varepsilon}, \theta^{\varepsilon}\right)(\tau)=(\tilde{\mathbf{u}}, \tilde{\nu}, \tilde{\theta})(\tau), \forall \tau \in[0, t]\right\}$. It is obvious that $\mathscr{K} \ni 0$. We prove that $\mathscr{K}$ is both open and closed in $\left[0, T_{1}\right]$, which then implies that $\mathscr{K}=\left[0, T_{1}\right]$.

Suppose $t_{0} \in \mathscr{K}$. Then the solution $\left(\mathbf{u}^{\varepsilon}, \nu^{\varepsilon}, \theta^{\varepsilon}\right)$ of the regularized problem exists in some interval $\left(0, t_{0}+\delta\right)$. Indeed, let us consider the Eqs. $(37),(38),(41)$ with standard boundary conditions and initial 
conditions $\left(\mathbf{u}^{\varepsilon}, \nu^{\varepsilon}, \theta^{\varepsilon}\right)\left(t_{0}\right)=(\tilde{\mathbf{u}}, \tilde{\nu}, \tilde{\theta})\left(t_{0}\right)$. Thus, in the open interval $\left(t_{0}, t_{0}+\delta\right)$ the new problem has a solution that is a continuation of $\left(\mathbf{u}^{\varepsilon}, \nu^{\varepsilon}, \theta^{\varepsilon}\right)$.

Next, we show that $\left(\mathbf{u}^{\varepsilon}, \nu^{\varepsilon}, \theta^{\varepsilon}\right)$ and $(\tilde{\mathbf{u}}, \tilde{\nu}, \tilde{\theta})$ coincide on some open interval. Consider Eqs. (37), (38) in the domain $Q_{t_{0}}$. Since $\mathbf{u}^{\varepsilon} \in L_{2}\left(\left(0, t_{0}\right) ; W_{\infty}^{1}(\Omega)\right)$, we can apply Theorem 4.1 (all we need from this theorem is the existence of the triple $(\mathbf{u}, \boldsymbol{\nu}, \mathbf{n})$ satisfying Eqs. (10), (11) and this fact does not depend on any statement below); thus $\theta^{\varepsilon}=\theta_{1}, \nu^{\varepsilon}=0$ in an $\alpha$-neighborhood of $\partial \Omega$ if $0<t<T^{\prime}$ for some constant $T^{\prime} \leq t_{0}$. This constant does not depend on $\varepsilon$ since $\mathbf{u}^{\varepsilon}=\tilde{\mathbf{u}}$ for any $t<t_{0}$ and $\|\tilde{\mathbf{u}}\|_{L_{2}\left(\left(0, t_{0}\right) ; W_{\infty}^{1}(\Omega)\right)}$ is also $\varepsilon$-independent by Proposition 3.1. Due to the definition of $T_{1}$, we have $\alpha>\frac{d}{3}$ and $T^{\prime}=t_{0}$.

Moreover, since $\mathbf{u}^{\varepsilon} \in L_{2}\left(\left(0, t_{0}+\delta\right) ; W_{\infty}^{1}(\Omega)\right)$ and $\left.\theta^{\varepsilon}\right|_{t=t_{0}}=\theta_{1},\left.\nu^{\varepsilon}\right|_{t=t_{0}}=0$ in the $\alpha$-neighborhood of $\partial \Omega$, there exists a constant $\delta_{\varepsilon}$ depending on $\varepsilon$ such that $\theta^{\varepsilon}=\theta_{1}, \nu^{\varepsilon}=0$ in the $\frac{d}{3}$-neighborhood of $\partial \Omega$ if $0<t<t_{0}+\delta_{\varepsilon}$.

Now it is easy to check that the solution $\left(\mathbf{u}^{\varepsilon}, \nu^{\varepsilon}, \theta^{\varepsilon}\right)$ satisfies the Eqs. (41), (49), (50) in $Q_{T_{1}}$, and hence coincides with $(\tilde{\mathbf{u}}, \tilde{\nu}, \tilde{\theta})$ in some neighborhood of $t=t_{0}$. Consequently, $\mathscr{K}$ is open.

We now show that $\mathscr{K}$ is closed, i.e., if $t_{n} \in \mathscr{K}$ and $t_{n} \rightarrow t_{0}$ then $t_{0} \in \mathscr{K}$. Indeed, $t_{0} \notin \mathscr{K}$ only if the $W_{2}^{2} \times W_{2}^{2} \times W_{2}^{3}$-norm of the solution $\left(\mathbf{u}^{\varepsilon}, \nu^{\varepsilon}, \theta^{\varepsilon}\right)$ at the point $t_{n}$ tends to $\infty$. This is impossible since we have inequalities (47), (48) for the solution $(\tilde{\mathbf{u}}, \tilde{\nu}, \tilde{\theta})$. Hence $t_{0} \in \mathscr{K}$.

Therefore, $\mathscr{K}$ is both open and closed in $\left[0, T_{1}\right]$ which implies that $\mathscr{K}=\left[0, T_{1}\right]$ and concludes the proof of the lemma.

Continuing the proof of Theorem 3.1, select a subsequence $\varepsilon_{k}$ such that $(\mathbf{u}, \boldsymbol{\nu}, \mathbf{n})$ is a weak limit of $\left(\mathbf{u}^{\varepsilon_{k}}, \boldsymbol{\nu}^{\varepsilon_{k}}, \mathbf{n}^{\varepsilon_{k}}\right)$ in the spaces $L_{2}\left((0, T) ; \stackrel{\circ}{S o l} l_{2}^{2}(\Omega)\right), L_{\infty}\left((0, T) ; \stackrel{\circ}{W_{2}^{1}}(\Omega)\right)$, and $L_{\infty}\left((0, T) ; W_{2}^{2}(\Omega)\right)$. The vector fields $(\mathbf{u}, \boldsymbol{\nu}, \mathbf{n})$ are the first three terms of the solution. The term $\nabla p^{\prime}$ is the Hodge projection of $\dot{\mathbf{u}}-\mu \Delta \mathbf{u}-\Delta \mathbf{n} \nabla \mathbf{n} \in L_{2}\left(Q_{T}\right)$ on the orthogonal complement of $L_{2}\left(0, T ; \operatorname{Sol}_{2}(\Omega)\right)$.

As in Sect. 2.5, one checks that the initial conditions hold.

The proof of uniqueness is identical to that in Sect. 2.6.

Remark 3.1. As can be seen from the proof, all that is needed is that $\mathbf{n}_{1}$ is piecewise constant on the boundary $\partial \Omega$.

The same result holds if external forces are present.

Theorem 3.2. Suppose $\Omega, \mathbf{n}_{0}, \boldsymbol{\nu}_{0}, \mathbf{u}_{\mathbf{0}}, \mathbf{n}_{1}$ satisfy the conditions of Theorem 3.1. Assume also that $\mathbf{F} \in$ $L_{2}\left((0, T) ; W_{2}^{1}(\Omega)\right), \mathbf{G}=\left(G_{1}, G_{2}, 0\right) \in L_{1}\left((0, T) ; W_{2}^{2}(\Omega)\right), F^{3}=0$. Then the solution exists and is unique for some $T>0$.

Remark 3.2. If $\mathbf{G} \neq\left(G_{1}, G_{2}, 0\right)$, the director field cannot be represented as $\mathbf{n}=(\cos \theta, \sin \theta, 0)$.

Remark 3.3. The analogues of the Theorems 3.1 and 3.2 hold also in the case of the director field

$$
\mathbf{n}=\left(\cos \theta \sin \phi_{0}, \sin \theta \sin \phi_{0}, \cos \phi_{0}\right), \quad \boldsymbol{\nu}=\left(0,0, \nu_{3}\right),
$$

where $\phi_{0}$ is a constant angle and $\theta, \nu_{3}$ are new unknown functions.

\section{Finite Propagation Speed}

In this section we consider the strong solutions of the Ericksen-Leslie equation both in the periodic (see Definition 2.1) and the bounded domain case (see Definition 3.1). For simplicity in this section we will use

$$
\int f d x_{1} d x_{2} \text { instead of } \int_{\mathbb{T}} f d x_{1} d x_{2} \text { or } \int_{\Omega} f d x_{1} d x_{2}
$$

depending on the nature of the domain. 
Theorem 4.1. Consider the Eqs. (10), (11), suppose that $w^{i j}:=u_{x_{i}}^{j}+u_{x_{j}}^{i}$ satisfy

$$
\left\|\underset{x}{\operatorname{esssup}}\left|w^{i j}(x, t)\right|\right\|_{L_{\alpha}(0, T)} \leq M / 2
$$

for some $1<\alpha \leq \infty$, and that $\|\mathbf{u}\|$ is bounded by a constant $m>0$. Assume also that $\nabla \mathbf{n}_{0}$ and $\boldsymbol{\nu}_{0}$ vanish for $\left\|x-x_{0}\right\|<r$. Then $\nabla \mathbf{n}$ and $\boldsymbol{\nu}$ are equal to zero for

$$
\left\|x-x_{0}\right\|<r-(m+\max \{1, K / J\}) t, \quad M t^{\frac{\alpha-1}{\alpha}}<\frac{1}{2} .
$$

In the case of a bounded domain $\Omega$, we impose the additional assumption $\left.u\right|_{\partial \Omega}=0, \boldsymbol{\nu}(x, t)=0$, $\mathbf{n}(x, t)_{t}=0$ if $(x, t) \in\left\{x \in \partial \Omega \mid\left\|x-x_{0}\right\|<r-t(m+\max \{1, K / J\})\right\}$.

Proof. Let $x_{0}=0$. Taking $\boldsymbol{\zeta}=\boldsymbol{\nu} \varphi$ and $\boldsymbol{\psi}=-K \Delta \mathbf{n} \varphi$ in (28) we get

$$
\begin{aligned}
& \left.\frac{J}{2} \int\|\boldsymbol{\nu}\|^{2} \varphi d x_{1} d x_{2}\right|_{0} ^{t}=\int\left(\frac{J}{2}\left(\varphi_{t}+u^{j} \varphi_{x_{j}}\right)\|\boldsymbol{\nu}\|^{2}-K(\Delta \mathbf{n} \times \mathbf{n}) \cdot \boldsymbol{\nu} \varphi\right) d x_{1} d x_{2} d t \\
& \left.\int \frac{K}{2}\|\nabla \mathbf{n}\|^{2} \varphi d x_{1} d x_{2}\right|_{0} ^{t}=\int\left(\frac{K}{2}\left(\varphi_{t}+u^{j} \varphi_{x_{j}}\right)\|\nabla \mathbf{n}\|^{2}-K(\boldsymbol{\nu} \times \mathbf{n}) \cdot \Delta \mathbf{n} \varphi\right. \\
& \left.\quad-K u_{x_{k}}^{j} \mathbf{n}_{x_{j}} \cdot \mathbf{n}_{x_{k}} \varphi-K \dot{\mathbf{n}} \cdot \mathbf{n}_{x_{j}} \varphi_{x_{j}}\right) d x_{1} d x_{2} d t .
\end{aligned}
$$

Add the previous two identities and rewrite the result as

$$
\begin{aligned}
& \left.\frac{J}{2} \int\|\boldsymbol{\nu}\|^{2} \varphi d x_{1} d x_{2}\right|_{0} ^{t}+\left.\int \frac{K}{2}\|\nabla \mathbf{n}\|^{2} \varphi d x_{1} d x_{2}\right|_{0} ^{t} \leq \int \dot{\varphi}\left(\frac{J}{2}\|\boldsymbol{\nu}\|^{2}+\frac{K}{2}\|\nabla \mathbf{n}\|^{2}\right) \\
& \quad+K \max _{i, j, x} w^{i j}\|\nabla \mathbf{n}\|^{2} \varphi+K\|\boldsymbol{\nu}\|\|\nabla \mathbf{n}\|\|\nabla \varphi\| d x_{1} d x_{2} d t .
\end{aligned}
$$

Denote $\varphi=\phi\left(\|x\|+m^{\prime} t\right)$, where $\phi(x) \in C^{1}(\mathbb{R}), \phi=0$ for $\|x\|>r, \phi^{\prime} \leq 0$. Let $m^{\prime}:=m+\max \{1, K / J\}$ and estimate

$$
\begin{gathered}
\int\left(\dot{\varphi}\left(\frac{J}{2 K}\|\boldsymbol{\nu}\|^{2}+\frac{1}{2}\|\nabla \mathbf{n}\|^{2}\right)+\|\boldsymbol{\nu}\|\|\nabla \mathbf{n}\|\|\nabla \varphi\|\right) d x_{1} d x_{2} d t \\
\leq \int \phi^{\prime}\left(\|x\|+m^{\prime} t\right)\left(m^{\prime}+\frac{u^{i} x_{i}}{\|x\|}\right)\left(\frac{J}{2 K}\|\boldsymbol{\nu}\|^{2}+\frac{1}{2}\|\nabla \mathbf{n}\|^{2}\right) \\
+\frac{\left|\phi^{\prime}\left(\|x\|+m^{\prime} t\right)\right|}{2}\left(\|\boldsymbol{\nu}\|^{2}+\|\nabla \mathbf{n}\|^{2}\right) d x_{1} d x_{2} d t \leq 0 .
\end{gathered}
$$

Consequently,

$$
\begin{aligned}
\frac{J}{2 K}\left\|\boldsymbol{\nu}(t) \phi^{\frac{1}{2}}\right\|_{2}^{2}+\frac{1}{2}\left\|\phi^{\frac{1}{2}} \nabla \mathbf{n}(t)\right\|^{2} & \leq \int \max _{i, j, x} w^{i j}\|\nabla \mathbf{n}\|^{2} \varphi d x_{1} d x_{2} d t \\
& \leq M t^{\frac{\alpha-1}{\alpha}} \underset{t}{\operatorname{esssup}}\left\|\phi^{\frac{1}{2}} \nabla \mathbf{n}\right\|_{2}^{2},
\end{aligned}
$$

which proves the statement.

Remark 4.1. The proof of Theorem 4.1 is independent of the existence and uniqueness proof and result. Moreover, in the proof we can suppose $(\mathbf{u}, \boldsymbol{\nu}, \mathbf{n})$ to satisfy only (10) and (11) but not (9).

Acknowledgments. GAC was partially supported by RFBR Grant 15-01-07920. TSR was partially supported by the NCCR SwissMAP Grant of the Swiss NSF. All authors were partially supported by the Government grant of the Russian Federation under the Resolution No. 220 "On measures designed to attract leading scientists to Russian institutions of higher education" according to the Agreement No. 11.G34.31.0054, signed by the Ministry of Education and Science of the Russian Federation, the leading scientist, and Lomonosov Moscow State University (on the basis of which the present research is organized). 


\section{References}

[1] de Gennes, P.G., Prost, J.: The physics of liquid crystals. Clarendon Press, Oxford (1993)

[2] Chechkin, G.A., Ratiu, T.S., Romanov, M.S., Samokhin, V.N.: Nematic liquid crystals. Existence and uniqueness of periodic solutions to Ericksen-Leslie equations. Bull. Ivan Fedorov Moscow State Univ. Print. Art 12, 139-151 (2012)

[3] Ratiu, T.S., Romanov, M.S., Chechkin, G.A.: Homogenization of the equations of the dynamics of nematic liquid crystals with inhomogeneous density. J. Math. Sci. 186(2), 322-329 (2012) [Translated from Problemy Mat. Analiza 66, 167-173 (2012)]

[4] Chechkin, G.A., Chechkina, T.P., Ratiu, T.S., Romanov, M.S.: Nematodynamics and random homogenization. Appl. Anal. doi:10.1080/00036811.2015.1036241 (2015, Online first)

[5] Ericksen, J.: Conservation laws for liquid crystals. Trans. Soc. Rheol. 5, 22-34 (1961)

[6] Ericksen, J.: Hydrostatic theory of liquid crystals. Arch. Ration. Mech. Anal. 9, 371-378 (1962)

[7] Leslie, F.: Some constitutive equations for anisotropic fluids. Q. J. Mech. Appl. Math. 19, 357-370 (1966)

[8] Leslie, F.: Some constitutive equations for liquid crystals. Arch. Ration. Mech. Anal. 28, 265-283 (1968)

[9] Ericksen, J.: Continuum theory of nematic liquid crystals. Res. Mech. 21, 381-392 (1987)

[10] Leslie, F.: Continuum theory for nematic liquid crystals. Continuum Mech. Thermodyn. 4(3), 167-175 (1992)

[11] Chandrasekhar, S.: Liquid crystals, 2nd edn. Cambridge Univ. Press, Cambridge (1992)

[12] Lin, F.-H., Liu C.: Existence of solutions for the Ericksen-Leslie system. Arch. Rat. Mech. Anal. 154(2), 135-156 (2000). doi:10.1007/s002050000102

[13] Gay-Balmaz, F., Ratiu, T.S.: The geometric structure of complex fluids. Adv. Appl. Math. 42, 176-275 (2009)

[14] Lin, F.H.: Nonlinear theory of defects in nematic liquid crystal: phase transition and flow phenomena. Commun. Pure Appl. Math. 42, 789-814 (1989)

[15] Lin, F.H., Liu, C.: Nonparabolic dissipative system modeling the ow of liquid crystals. Commun. Pure Appl. Math. XLVIII, 501-537 (1995)

[16] Shkoller, S.: Well-posedness and global attractors for liquid crystals on Riemannian manifolds. Commun. Partial Differ. Equ. 27, 1103-1137 (2002)

[17] Hong, M.C.: Global existence of solutions of the simplified Ericksen-Leslie system in dimension two. Calc. Var. Partial Differ. Equ. 40, 15-36 (2011)

[18] Lin, F.H., Liu, J.Y., Wang, C.Y.: Liquid crystal flows in two dimensions. Arch. Rat. Mech. Anal. 197, 297-336 (2010)

[19] Hong, M.C., Xin, Z.P.: Global existence of solutions of the liquid crystal ow for the Oseen-Frank model in $\mathbb{R}^{2}$. Adv. Math. 231, 1364-1400 (2012)

[20] Wang, C.Y.: Well-posedness for the heat flow of harmonic maps and the liquid crystal flow with rough initial data. Arch. Ration. Mech. Anal. 200, 1-19 (2011)

[21] Jiang, F., Jiang, S., Wang, D.: Global weak solutions to the equations of compressible flow of nematic liquid crystals in two dimensions. Arch. Rational Mech. Anal. 214, 403-451 (2014)

[22] Hieber, M., Nesensohn, M., Prüss, J., Schade, K.: Dynamics of nematic liquid crystal flows: the quasilinear approach. Ann. Inst. H. Poincaré, Analyse Nonlinéaire (2014, to appear). doi:10.1016/j.anihpc.2014.11.001

[23] Ratiu, T.S., Romanov, M.S., Samokhin, V.N., Chechkin, G.A.: Existence and uniqueness theorems in two-dimensional nematodynamics. Finite speed of propagation. Dokl. Akad. Nauk 462(5), 519-523 (2015) [English translation Doklady Mathematics 91(3), 354-358 (2015)]

[24] Ladyzhenskaya, O., Uraltseva, N.: Linear and quasilinear elliptic equations. Academic Press, New York (1968)

[25] Sobolev, S.L.: Some applications of functional analysis in mathematical physics, 3rd edn. Translations of Mathematical Monographs Series, vol. 90. AMS Press, Providence, Rhode Island (1991)

[26] Mikhailov V.P.: Partial differential equations. Mir (1978)

Gregory A. Chechkin and Maxim S. Romanov

Department of Differential Equations

Faculty of Mechanics and Mathematics

M.V. Lomonosov Moscow State University

Moscow 119991

Russia

e-mail: chechkin@mech.math.msu.su

Maxim S. Romanov

e-mail: mcliz@mail.ru

Tudor S. Ratiu

Department of Mathematics

Jiao Tong University

800 Dongchuan Road

Minhang, 200240 Shanghai

China
Tudor S. Ratiu

Section de Mathématiques

École Polytechnique Fédérale de Lausanne

1015 Lausanne, Switzerland

e-mail: tudor.ratiu@epfl.ch

Vyacheslav N. Samokhin

Moscow State University of Printing Art 2A

Pryanishnikova ul., Moscow 127550, Russia

e-mail: vnsamokhin@mtu-net.ru 